\title{
Boltzmann equation with double-well potentials
}

\author{
Silvia Chiacchiera, ${ }^{1, *}$ Tommaso Macrì, ${ }^{2,3}$ and Andrea Trombettoni ${ }^{4,5}$ \\ ${ }^{1}$ CFisUC, Department of Physics, University of Coimbra, P-3004-516 Coimbra, Portugal \\ ${ }^{2}$ Departamento de Física Teórica e Experimental, Universidade Federal do Rio Grande do Norte, 59072-970 Natal-RN, Brazil \\ ${ }^{3}$ International Institute of Physics, 59078-400 Natal-RN, Brazil \\ ${ }^{4}$ CNR-IOM DEMOCRITOS Simulation Center, Via Bonomea 265, I-34136 Trieste, Italy \\ ${ }^{5}$ SISSA and INFN, Sezione di Trieste, Via Bonomea 265, I-34136 Trieste, Italy
}

(Received 6 February 2016; published 26 October 2016)

\begin{abstract}
We study the dynamics of an interacting classical gas trapped in a double-well potential at finite temperature. Two model potentials are considered: a cubic box with a square barrier in the middle, and a harmonic trap with a Gaussian barrier along one direction. The study is performed using the Boltzmann equation, solved numerically via the test-particle method. We introduce and discuss a simple analytical model that allows one to provide estimates of the relaxation time, which are compared with numerical results. Finally, we use our findings to make numerical and analytical predictions for the case of a fermionic mixture in the normal-fluid phase in a realistic double-well potential relevant for experiments with cold atoms.
\end{abstract}

DOI: 10.1103/PhysRevA.94.043643

\section{INTRODUCTION}

Double-well energy potentials, with two, degenerate or not, minima separated by a maximum, are ubiquitous in several branches of science. These potentials are used to model a variety of processes involving an energy barrier [1], from the computation of rate coefficients in a chemical reaction [2] and the modeling of solid-state junctions [3] to the calculations involving nonperturbative instanton configurations in quantum field theory [4]. The importance per se of the study of the dynamics in double-wells potentials stems also from the fact that it is often preliminary to (and useful for) the investigation of more complex dynamical effects in multiwell potentials.

The prototypical problem of the dynamics in a double-well potential is to determine in how much time the particles move from one well to the other one and if (and in how much time) they equilibrate reaching a vanishing $\Delta N$, where $\Delta N$ is the difference of population between the two wells. A source of interwell motion is of course given by the quantum tunneling $[3,5]$. When quantum effects are dominant, a single particle tunnels from one well to the other, and for many particles the macroscopic quantum coherence exhibited at low temperature by many systems-including ${ }^{4} \mathrm{He}$ and ${ }^{3} \mathrm{He}$, ultracold atoms, and superconductors [6,7]—allows for a net current between the wells. At variance, at high temperatures thermal effects give rise to noise-assisted hopping events [1]: for ultracold atoms, at temperatures higher than the temperature at which the effects of quantum statistics become relevant, this corresponds to an incoherent flow of atoms and the ensuing thermalization of the two-well systems with $\Delta N \rightarrow 0$ (for a study of a polarized Fermi gas in a 3D double-well potential see [8]).

Experiments with cold atoms in double-well potentials give the concrete possibility to explore physical situations in which both phenomena-quantum tunneling and thermal noise-assisted hoppings - are present. The high degree of control of atomic gases at very low temperatures [9] allows

\footnotetext{
*Present address: Science and Technology Facilities Council (STFC), Daresbury Laboratory, Keckwick Lane, Daresbury, Warrington WA4 4AD, United Kingdom.
}

one to design and perform experiments where particles, either bosons, fermions, or mixtures of both, are subjected to properly engineered and highly tunable trapping potentials. In this context, superfluid double- and multiwell dynamics have been extensively studied, both theoretically and experimentally, primarily for bosonic atoms and more recently also for fermionic gases. For bosons in the superfluid regime at $T=0$, which are well described by the Gross-Pitaevskii equation, this has lead to the identification of the atomic analog of the Josephson effect of superconductivity and of the macroscopic quantum self-trapping effect [10-14], a direct consequence of the nonlinearity of the dynamical equations of motion. The study of superfluid fermions in double-well potentials is more recent and experiments for these systems are in progress. The peculiarity of such fermionic systems is, among others, the tunability of the strength of the interparticle interactions via the so-called Feshbach resonances, which results in the well known BCS-BEC crossover [15]. In a recent experiment [16] Valtolina et al. studied ultracold fermionic ${ }^{6} \mathrm{Li}$ atoms in two different hyperfine states loaded in double-well potentials, reporting on the observation of the Josephson effect between fermionic superfluids along the crossover.

Regarding thermal effects, another class of experiments focused during the years on the study of (i) polarized and twocomponent fermionic gases across and above the transition from the superfluid to the normal state occurring at the critical temperature $T_{c}$, and (ii) bosons at finite temperature, also near and above the Bose-Einstein condensation temperature $T_{\mathrm{BEC}}$ ( $T_{\mathrm{BEC}}$ coinciding with $T_{c}$ for Bose-Einstein condensates). The Boltzmann equation [17] provides a major tool to describe the collective dynamics of cold atoms in the normal-fluid phase [18-26] where one can extract the characteristic oscillation frequency and the damping of the various modes. Very recently undamped monopole breathing collective oscillations were observed in a 3D classical gas of ${ }^{87} \mathrm{Rb}$ atoms in agreement with the prediction of the Boltzmann equation [27]. Interestingly more recent analytical developments lead to new exact solutions of the Boltzmann equation in time-dependent potentials which may serve to engineer dynamical protocols to connect nonadiabatically two different equilibrium states [28]. 
Quite generally from studies of collective oscillations it emerged that the description with the Boltzmann equation (including the quantum statistics modification of the collision term [29]) works rather well not only for fermions above the Fermi temperature $T_{F}$ and for bosons above $T_{\mathrm{BEC}}$, but also for two-components fermions at temperature smaller than $T_{F}$ and larger than $T_{c}$ [30-33]. We also mention that the dynamics of bosons at finite temperature below $T_{\mathrm{BEC}}$ has been studied resorting to a Boltzmann-like description of the thermal part of the gas [34] — a similar approach based on the Boltzmann description of the nonsuperfluid part of a two-component fermionic mixture has been discussed in [35]. Most of these studies have been performed in single-well potentials without tunneling between wells, also given the difficulty of computing in a quantitatively reliable way the relaxation time $\tau$ which is determined at finite temperature by rare events of hopping across the wells. Studies of the dynamics of a Bose gas below $T_{\mathrm{BEC}}$ in a periodic multiwell potential were presented in [36-39], while the study of the fermionic transport in optical lattices at finite temperature (above $T_{c}$ ) was reported in [40], where the Boltzmann equation was investigated in local relaxation time approximation. The center-of-mass oscillations of a normal Fermi gas in a one-dimensional periodic potential were studied both theoretically and experimentally [41,42].

Although from one side a huge effort has been devoted to the study of quantum tunneling for superfluid atomic gases in double-well potentials at low temperatures, and from the other side the study of reaction-rate theory [1] and of the Boltzmann equation are two workhorses of nonequilibrium physics, the study of the Boltzmann equation itself in a doublewell potential is to the best of our knowledge a relatively not addressed topic. Motivated by systems of cold atoms in tunable geometries at finite (and possibly) variable temperature, in this paper we therefore study the double-well dynamics of an interacting gas at finite temperature within the framework of the Boltzmann equation with the classical collision term.

Our aim is to understand and describe, both qualitatively and quantitatively, the effect of two-body collisions on the double-well dynamics. This problem is interesting in view of current and future experiments with cold atoms at finite temperature, since our approach can be applied to study the normal-fluid dynamics in double-well potentials. Here we examine how a gas in a symmetric double well, prepared with an initial population imbalance, relaxes towards the numberbalanced equilibrium and we propose a simple analytical model to describe our numerical findings. Performing a comparison between numerical and analytical results, we analyze the relaxation time as a function of barrier heights and interaction strengths. We observe that, even though we are not going to consider the effect of the quantum statistics on the collision term, we expect that both the numerical computations and the analytical model can be straightforwardly extended to include such correction and that the relaxation time would have a similar dependence on barrier heights and interaction strengths, with qualitative changes of the dependence of $\tau$ on the temperature only for, say, $T / T_{F} \lesssim 0.5[30,31]$ (and therefore close to $T_{c}$ in the unitary limit).

The article is organized as follows. In Sec. II we introduce the formalism briefly describing the numerical method used in the work. We also define the two model double-well potentials we consider. The first model is a square-well potential with a barrier of vanishing width in the middle that provides a simplified toy model for our numerical and analytical studies. The second model, which is directly inspired by experimental work with cold atoms, is the superposition of a harmonic isotropic potential and a Gaussian barrier. In Sec. III we introduce a simple analytical model for the study of the dynamics. In Sec. IV and Sec. V we present the numerical results for, respectively, the square double-well potential and the realistic one and compare them with the predictions of the analytical model. Finally, we draw in Sec. VI our conclusions and discuss possible improvements of the present paper for future work, while some useful results are collected in the Appendixes.

\section{BOLTZMANN EQUATION WITH A DOUBLE-WELL POTENTIAL}

In this section we briefly recall the Boltzmann equation and introduce the two double-well potentials we consider in the following. Section II A is devoted to introduce the Boltzmann equation formalism and recall some of its basic properties used hereafter, while in Sec. II B we briefly sketch the procedure for the numerical solution of the Boltzmann equation based on the test-particle method. Section II C is devoted to introducing the two double-well potentials studied in this paper: a "toy" square double well (SDW) with a filtering wall and a more realistic harmonic-Gaussian double-well (HGDW) potential. In Sec. II D we define the various time scales appearing in the double-well problem.

\section{A. Boltzmann equation}

We consider a one-component gas of $N$ classical interacting particles of mass $m$, in an external potential $V(\mathbf{r})$. The evolution in time of the phase-space distribution function $f(\mathbf{r}, \mathbf{p}, t)$ is governed by the Boltzmann equation [17]

$$
\frac{\partial f}{\partial t}+\dot{\mathbf{r}} \cdot \nabla_{\mathbf{r}} f+\dot{\mathbf{p}} \cdot \nabla_{\mathbf{p}} f=-I[f] .
$$

In the left-hand side $\dot{\mathbf{r}}=\mathbf{p} / m$ and $\dot{\mathbf{p}}=-\nabla_{\mathbf{r}} V$, while in the right-hand side $I[f]$ is the collision integral

$$
I[f]=\int \frac{d^{3} p_{1}}{(2 \pi \hbar)^{3}} d \Omega \frac{d \sigma}{d \Omega} \frac{\left|\mathbf{p}-\mathbf{p}_{1}\right|}{m}\left(f f_{1}-f^{\prime} f_{1}^{\prime}\right),
$$

where $\frac{d \sigma}{d \Omega}$ is the differential cross section and the notation $f, f_{1}, f^{\prime}, f_{1}^{\prime}$ is used as a shortcut for the distribution function evaluated at the same $\mathbf{r}, t$, but with momenta $\mathbf{p}, \mathbf{p}_{1}, \mathbf{p}^{\prime}, \mathbf{p}_{1}^{\prime}$, respectively. The normalization condition is $\int d \Gamma f=N$, and the average of any one-body variable $\mathcal{O}(\mathbf{r}, \mathbf{p})$ is $\langle\mathcal{O}\rangle=$ $\frac{1}{N} \int d \Gamma f \mathcal{O}$, where the phase-space volume element is $d \Gamma=$ $d^{3} r d^{3} p /(2 \pi \hbar)^{3}$.

At equilibrium, the distribution function reads

$$
f_{\mathrm{eq}}(\mathbf{r}, \mathbf{p})=e^{-\beta\left(\frac{p^{2}}{2 m}+V(\mathbf{r})-\mu\right)},
$$

where $\beta=1 /\left(k_{B} T\right)$ is the inverse temperature and $\mu$ the chemical potential. The total collision rate at equilibrium can 
be computed exactly using

$$
\Gamma_{\mathrm{eq}}=\frac{1}{2} \int d^{3} r \int \frac{d^{3} p}{(2 \pi \hbar)^{3}} \frac{d^{3} p_{1}}{(2 \pi \hbar)^{3}} d \Omega \frac{d \sigma}{d \Omega} \frac{\left|\mathbf{p}-\mathbf{p}_{1}\right|}{m} f_{\mathrm{eq}} f_{1, \mathrm{eq}},
$$

where the factor $1 / 2$ is needed to avoid double counting (since we are dealing with a one-component gas). Expressions for the collision rate in a single square well and in a harmonic potential are collected in Appendix A.

In this work, we consider particles interacting with a constant cross section, i.e., with no energy or momentum dependence:

$$
\frac{d \sigma}{d \Omega}=\frac{\sigma}{4 \pi}
$$

\section{B. Test-particle method}

A fully numerical approach to solve the Boltzmann equation is provided by the so-called test-particle method, in which the coordinates of all the particles in phase space are evolved individually. This method, similar to molecular dynamics but with a stochastic component, was developed in the ' 80 s in the context of nuclear physics [43] and recently has been applied also to cold atoms [26,33,44-47]. Formally, the distribution function $f(\mathbf{r}, \mathbf{p}, t)$ is discretized as a sum of $\delta$ functions peaked at the position and momentum of each test particle:

$$
f(\mathbf{r}, \mathbf{p}, t)=\frac{N}{\tilde{N}} \sum_{i=1}^{\tilde{N}}(2 \pi \hbar)^{3} \delta\left(\mathbf{p}-\mathbf{p}_{i}(t)\right) \delta\left(\mathbf{r}-\mathbf{r}_{i}(t)\right),
$$

where $N$ is the number of real particles and $\tilde{N}$ is the number of test particles, those entering the simulation. In some cases it is convenient to have $\tilde{N} \neq N$ : if the number of real particles is very low, one associates to each real particle many test particles $(\tilde{N}>N)$, to describe the evolution in phase space with a finer resolution. At variance, in the opposite case of too many real particles to be simulated individually, one test particle represents many real ones $(\tilde{N}<N)$ [33]. We observe that for a generic $N / \tilde{N}$, the interactions between test particles are ruled by a cross section that is related to the real one as follows:

$$
\tilde{\sigma}=\frac{N}{\tilde{N}} \sigma
$$

In this work, we always take $N=\tilde{N}$.

The average value of any one-body observable $\mathcal{O}(\mathbf{r}, \mathbf{p})$ is obtained as follows:

$$
\langle\mathcal{O}\rangle=\frac{1}{N} \int d \Gamma f(\mathbf{r}, \mathbf{p}, t) \mathcal{O}(\mathbf{r}, \mathbf{p})=\frac{1}{\tilde{N}} \sum_{i=1}^{\tilde{N}} \mathcal{O}\left(\mathbf{r}_{i}, \mathbf{p}_{i}\right) .
$$

In the absence of interparticle interactions, the phase-space coordinates of each test particle are evolved according to the Hamilton equations

$$
\dot{\mathbf{r}}=\frac{\mathbf{p}}{m}, \quad \dot{\mathbf{p}}=-\nabla_{\mathbf{r}} V(\mathbf{r}) .
$$

The actual numerical scheme to integrate them depends on the potential. In the case of a simple box, particles are propagated via the Euler method (exact in this case), and the collisions with the walls are implemented reversing the appropriate momentum component. For a generic potential, the particle position and momentum are evolved from the time step $t_{n}$ to $t_{n+1}=t_{n}+\Delta t$ via the velocity Verlet algorithm [48]

$$
\begin{aligned}
& \mathbf{v}\left(t_{n+\frac{1}{2}}\right)=\mathbf{v}\left(t_{n}\right)+\mathbf{a}\left(t_{n}\right) \Delta t / 2, \\
& \mathbf{r}\left(t_{n+1}\right)=\mathbf{r}\left(t_{n}\right)+\mathbf{v}\left(t_{n+\frac{1}{2}}\right) \Delta t, \\
& \mathbf{v}\left(t_{n+1}\right)=\mathbf{v}\left(t_{n+\frac{1}{2}}\right)+\mathbf{a}\left(t_{n+1}\right) \Delta t / 2,
\end{aligned}
$$

with $\mathbf{a}(t)=-\nabla V(\mathbf{r}(t)) / m$ the acceleration and $t_{n+\frac{1}{2}}$ an intermediate time step.

For interacting particles, collisions have to be implemented too. The cross section defines an interaction range $d_{\text {int }}=$ $\sqrt{\sigma / \pi}$ : in each time step, all the pair of particles (within a certain distance) are checked, and they are collided if (1) they reach their closest approach [46] in the time step and (2) their distance at closest approach is within the interaction range. A collision takes place randomly assigning new momenta to the participants, with the constraints of energy and momentum conservation. In our simulations the trajectories of the colliding particles are corrected to take into account the fact that the collision takes place at the closest approach point [46]. One can check that in the case of a harmonic well this correction is necessary to respect the balance of kinetic and potential energy. We have performed reliability tests of our code, similar to those presented in Ref. [46], to ensure that both propagation and collisions are correctly implemented.

We conclude this section by noticing that in the double-well case the correction of trajectories has a drawback, namely, some particles overcome the barrier even if they should not, and this leads to a bias flux from the more populated well to the other one. However, we have checked that the effect on the relaxation time is at most $\sim 20 \%$ (typically smaller), and we prefer to keep the correction of trajectories for the reason explained above. One could adopt a hybrid approach and accept only corrections that do not cause a "leak" of particles, but this would considerably slow down the simulation.

\section{Two models for the double well}

We consider two model potentials for the double well. The SDW potential has a rectangular energy barrier (of negligible width) located at the center of the system, which is in turn chosen to be a square well. This SDW has the advantage to be more simply numerically simulated using the test-particle method and it allows for an analytical treatment of the approximate model we are going to introduce in Sec. III for the determination of the relaxation times in double-well potentials. The other potential is relevant for cold-atom experiments and it is provided by the sum of a harmonic potential plus an energy barrier, chosen of Gaussian form both for simplicity and also because such a barrier could be created by a superimposed blue-detuned potential. We refer to the second double-well potential as the harmonic-Gaussian double-well (HGDW) potential.

The first model (SDW) is a toy model corresponding to a cubic box of side $2 L$ partitioned into two regions by a filtering wall: i.e., particles are allowed to pass through it or are reflected depending upon their momentum component orthogonal to the wall (say, $p_{x}$ ). When a particle during its propagation tries to 
cross the plane $x=0$, we check for its momentum along $x$ : if $\left|p_{x}\right|>p_{0}$, where $p_{0}=\sqrt{2 m V_{0}}$ and $V_{0}$ is the height of the barrier, then the particle it is allowed to pass, as if the barrier were not there; if instead $\left|p_{x}\right|<p_{0}$, it is reflected by the barrier. This model can be seen as a finite barrier of negligible thickness $w \rightarrow 0$, and the corresponding potential reads

$V(\mathbf{r})= \begin{cases}V_{0}\left(\theta\left(x+\frac{w}{2}\right)+\theta\left(\frac{w}{2}-x\right)-1\right), & |x|,|y|,|z| \leqslant L \\ \infty, & \text { else. }\end{cases}$

This model has the advantage to simplify both numerical and analytical approaches and it allows one to study the effect of the barrier without introducing a specific shape for it.

The second potential (HGDW) is more realistic and it represents an isotropic harmonic potential (i.e., a spherical trap) plus a Gaussian barrier in one direction:

$$
V(\mathbf{r})=\frac{m \omega_{0}^{2} r^{2}}{2}+\tilde{V} e^{\frac{-x^{2}}{2 w^{2}}}
$$

This is a realistic form of double-well potential in cold-atom experiments. In Fig. 1 we represent schematically the two model potentials at $z=0$, as a function of $x$ and $y$. In this pictorial representation, the vertical coordinate of the particles corresponds to their energy.

In both cases, we denote by $V_{0}$ the barrier height: it is $V_{0}=p_{0}^{2} / 2 m$ for the SDW, and $V_{0}=V(\mathbf{0})-V\left(\mathbf{r}_{\min }\right)$, the difference of potential energy between the barrier top and the well minima, in the case of the HGDW.

The quantity we use to follow the macroscopic dynamics of the double-well problem is the fraction of particles in the left well at time $t$,

$$
x_{L}(t)=\frac{N_{L}(t)}{N} .
$$

The population imbalance is defined as

$$
\Delta N(t)=N_{L}(t)-N_{R}(t) .
$$

In the literature, as in the case of the superfluid dynamics in double-well potentials, is also often used as well the relative imbalance $z(t)=\Delta N(t) / N$. Typically we choose $z(t=0)>0$.

\section{Time scales}

There are in general two fundamental time scales that rule the dynamics of a trapped interacting gas in a confining trap: one is related to the interparticle interactions, the other to the external potential.

The average time $\tau_{\text {coll }}$ between two consecutive collisions experienced by the same particle is the collisional time

$$
\tau_{\text {coll }}=\left(2 \Gamma_{\mathrm{eq}} / N\right)^{-1} .
$$

On the other hand, the average time $\tau_{\text {trap }}$ a particle needs to travel across the whole trap is

$$
\tau_{\text {trap }}=\frac{l_{\text {trap }}}{v_{\text {rms }}}
$$

where the root-mean-square velocity at equilibrium is $v_{\mathrm{rms}}=$ $\sqrt{3 /(\beta m)}$, and $l_{\text {trap }}$ the system linear size. The reference length $l_{\text {trap }}$ is chosen to be $2 L$ for the SDW model (and as well for the
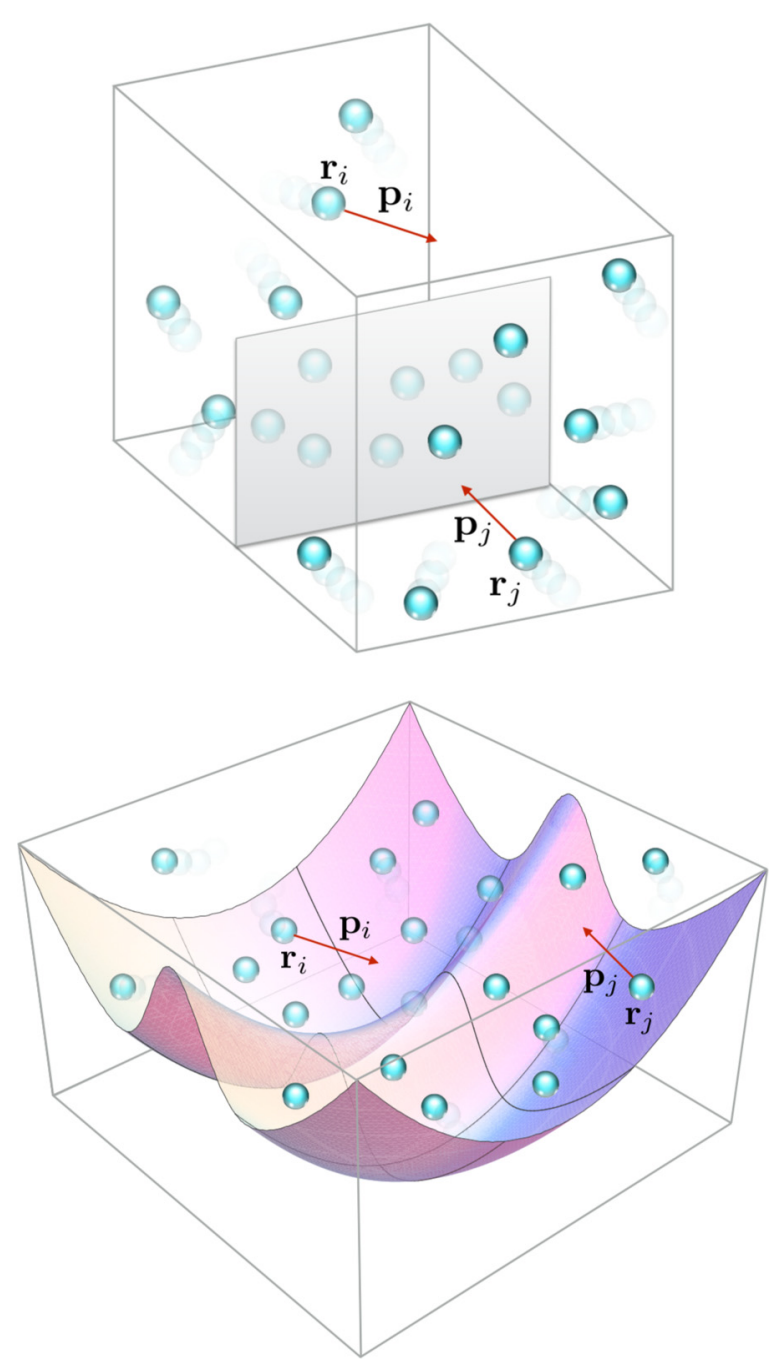

FIG. 1. Plot of the SDW (top) and HGDW (bottom) potentials. The figures represent $V(x, y, z=0)$, where $V$ is the potential energy. Particles are also pictorially shown, with their vertical coordinate representing their energy.

single square well treated in Sec. III A) and $2 \sqrt{2 \tilde{V} /\left(m \omega_{0}^{2}\right)}$ for the HGDW model (it is the distance between the two points of the harmonic trap having energy $\tilde{V}$, neglecting the barrier).

Depending upon the frequency of collisions, the gas can be in different dynamical regimes, the two limiting cases being the collisionless regime (very rare collisions) and the hydrodynamical one (very frequent collisions): if $\tau_{\text {coll }} / \tau_{\text {trap }} \rightarrow$ $\infty$ the gas is collisionless, whereas it is hydrodynamical if $\tau_{\text {coll }} / \tau_{\text {trap }} \rightarrow 0$.

For the double-well problem, we are interested in the time needed to smooth out the initial population imbalance, and our aim is to relate it to the basic properties of the system. This defines for double-well potentials a third time scale, related to the relaxation of imbalance, and that we will indicate with $\tau$. Classically, the particles can cross the barrier only if their momentum perpendicular to it is high enough. Since the momentum is continuously redistributed between particles in collisional events, we expect that the relaxation time depends on $\tau_{\text {coll }}$. Also, an Arrhenius type of behavior 
$1 / \tau \propto \exp \left(-\beta V_{0}\right)$ is expected in the limit of large barriers $\left(\beta V_{0} \gtrsim 1\right)$.

These three time scales $\left(\tau_{\text {coll }}, \tau_{\text {trap }}, \tau\right)$ will of course depend on the potential considered. Throughout the next sections, for different reasons, we deal with a square single well, a square double well, a harmonic oscillator, and a harmonic-Gaussian double well. To keep as light as we can the notation, we use the following convention: within any subsection, unless otherwise stated and denoted (respectively with $s w, S D W, H O$, and $H G D W$ ), we intend that the potential-dependent quantities are computed within the potential under consideration.

\section{SIMPLE ANALYTICAL MODEL}

In this section we develop a simple analytical model to describe the effect of collisions on the relaxation dynamics.

This model takes inspiration from the tight-binding ansatz used for the Gross-Pitaevskii equation in double- or multiwell potentials $[10,49,50]$. The Gross-Pitaevskii equation, describing the dynamics of a superfluid, is an equation for a complex wave function $\psi$ : one then introduces two degrees of freedom for $\psi$ (phase, $\varphi$, and number, $N$ ) and this leads for a double-well potential to the introduction of four degrees of freedom, two per well (say $\varphi_{L}, \varphi_{R}$ and $N_{L}, N_{R}$ in the left, $L$, and right, $R$, wells). These four degrees of freedom are not independent since the total number of particles $N_{L}+N_{R}$ is constant. Via these four nonindependent degrees of freedom one then obtains a simplified, yet very good, description of the superfluid tunneling dynamics in the weakly coupled regime.

Of course, for a classical gas in a double-well potential, there is no tunneling and the distribution $f$ is not a complex number: anyway, one can still think to introduce suitable degrees of freedom per well and after coupling the two wells via the Boltzmann equation one obtains a description of the double-well dynamics. This leads to a simplified model, which allows for an approximate estimate of the relaxation time $\tau$. It is the choice of the degrees of freedom in the separate wells (effectively one in the model below, or eventually more for a more accurate description) that characterizes the model. Such choice is suggested by the form of the potential and by the properties of the physical system at hand: as an example, in [51] a simplified model was introduced to study the nonequilibrium distribution functions for electrons in the electrodes of a metal-insulator-metal junction, while charge transfer and coherence dynamics of a tunnelling system coupled to an harmonic oscillator were studied in [52].

In our case, we proceed to a rather drastic modelization of the Boltzmann equation dynamics taking into account that it is the energy barrier $V_{0}$ that sets an energy scale, dividing particles in two classes: the particles having energy larger than $V_{0}$ (which then can move from one well to the other) and the particles having energy smaller than $V_{0}$ (which do not) [53]. The mechanism for which particles can move from the latter class to the former is provided by interactions: two particles scatter below barrier and as a consequence of the scattering one of the two, conserving energy, has an energy larger than $V_{0}$. Finer details, such as higher-order scattering processes, are neglected. As a result the model is not expected to give a quantitatively accurate prediction of the relaxation time. Nevertheless, since the estimate of the relaxation time is in general a not simple problem, and what in particular is difficult is the determination of prefactors, it provides simple analytical formulas which are in general qualitatively reasonable. In the regime of intermediate barriers $\left(\beta V_{0} \sim 1\right)$ the agreement is found to be also quantitative.

We start with the single-well problem: this allows one to classify the different types of collisions that will play a role in the double-well case and quantify their relative importance.

\section{A. Square single well}

Consider $N$ particles in a cubic box of volume $\Omega$ having size $2 L$. This is the same potential that will be considered in the next Sec. III B, except the fact that there a filtering wall is added in $x=0$.

In view of the double-well potential problem which we are interested in, we choose a reference momentum $p_{0}$ and a reference energy $V_{0}=p_{0}^{2} / 2 m$ (remember that here this choice is arbitrary since there is no barrier yet). We define $l_{\text {trap }} \equiv 2 L$ and $N^{>}(t)\left[N^{<}(t)\right]$ the number of particles of the gas having $\left|p_{x}\right|>p_{0}\left(\left|p_{x}\right|<p_{0}\right)$ at time $t$. Clearly, $N^{>}(t)+N^{<}(t)=N$ at any time, and, at equilibrium, collisions maintain them to their equilibrium values $N_{\text {eq }}^{\gtrless}$ (given in Appendix B). However, what is their evolution in time if the system starts from a situation with $N \gtrless \neq N_{\text {eq }}^{\gtrless}$ ?

We make the following assumption for the distribution function:

$$
f(\mathbf{p}, t)=g^{>}(\mathbf{p}) N^{>}(t)+g^{<}(\mathbf{p}) N^{<}(t),
$$

where

$$
g^{>}(\mathbf{p}) \equiv e^{-\beta\left(\frac{p^{2}}{2 m}-\mu^{>}\right)} \theta\left(p_{x}^{2}-p_{0}^{2}\right)
$$

and

$$
g^{<}(\mathbf{p}) \equiv e^{-\beta\left(\frac{p^{2}}{2 m}-\mu^{<}\right)} \theta\left(p_{0}^{2}-p_{x}^{2}\right) .
$$

$\beta$ equals $1 /\left(k_{B} T\right)$ where $T$ is the equilibrium temperature, and the chemical potentials $\mu \gtrless$ are constant (see Appendix B) and such that $\int d \Gamma g \gtrless(\mathbf{p})=1$, so that $\int d \Gamma f=N^{>}(t)+$ $N^{<}(t)=N$, as it should. Notice that the choice Eq. (17) implies a distribution that is uniform in space, thermal for $p_{y}$ and $p_{z}$, and has a discontinuity in the $p_{x}$ momentum distribution at $p_{x}^{2}=p_{0}^{2}$. Since the number of particles in the well is fixed there is only an independent parameter, e.g. $N^{>}(t)$, in Eq. (17) (given that the $g$ 's are fixed by $\beta$ and $V_{0}$ ).

Let us now consider, among all the possible collisions, those that will alter $N^{>}$and $N^{<}$. In a collision, each of the two incoming and outgoing particles can have momentum along $x$ above or below the reference values: 16 cases are therefore possible. Among these, there are six types of processes altering $N \gtrless$, namely

$$
\begin{array}{ll}
A:(<<;<>), & B:(<>;<<), \\
C:(>>;<>), & D:(<>;>>), \\
E:(<<;>>), & F:(>>;<<) .
\end{array}
$$

For example, with the notation $(<<;<>)$ we mean that in the collision the two incoming particles have $\left|p_{x}\right|<p_{0}$, whereas one of the outgoing ones (order does not matter) is above and one below reference. Let us indicate as $\Gamma_{i}, i=A, B, C, D, E, F$ 
the rates of each kind of process: the evolution in time of $N \gtrless$ satisfies

$$
\begin{aligned}
& \dot{N}^{>}(t)=\Gamma_{A}-\Gamma_{B}-\Gamma_{C}+\Gamma_{D}+2 \Gamma_{E}-2 \Gamma_{F}, \\
& \dot{N}^{<}(t)=-\Gamma_{A}+\Gamma_{B}+\Gamma_{C}-\Gamma_{D}-2 \Gamma_{E}+2 \Gamma_{F},
\end{aligned}
$$

and $\dot{N}^{>}(t)+\dot{N}^{<}(t)=0$, as it should. Each term $\Gamma_{i}$ is multiplied by an appropriate factor taking into account the changes in $N \gtrless$ the process implies. By a direct computation of the rates it is found that

$$
\begin{aligned}
\Gamma_{A}=\gamma_{1}\left(r^{<}\right)^{2} ; & \Gamma_{B}=\gamma_{1} r^{>} r^{<}, \\
\Gamma_{C}=\gamma_{2}\left(r^{>}\right)^{2} ; & \Gamma_{D}=\gamma_{2} r^{>} r^{<}, \\
2 \Gamma_{E}=\gamma_{3}\left(r^{<}\right)^{2} ; & 2 \Gamma_{F}=\gamma_{3}\left(r^{>}\right)^{2},
\end{aligned}
$$

where $r \gtrless(t) \equiv N \gtrless(t) / N_{\text {eq }}^{\gtrless}$. At equilibrium, $r_{\text {eq }}^{\gtrless}=1$ and the rates of the processes just defined are equal two by two: $\Gamma_{A}^{\mathrm{eq}}=$ $\Gamma_{B}^{\mathrm{eq}}, \Gamma_{C}^{\mathrm{eq}}=\Gamma_{D}^{\mathrm{eq}}$, and $\Gamma_{E}^{\mathrm{eq}}=\Gamma_{F}^{\mathrm{eq}}$. The factors $\gamma_{i}, i=1,2,3$ are appropriate equilibrium phase-space integrals detailed in Appendix C. They are proportional to the equilibrium collision rate: in fact, they are given by

$$
\gamma_{i}=\Gamma_{\mathrm{eq}} h_{i}\left(\beta V_{0}\right), \quad i=1,2,3,
$$

where $V_{0}=p_{0}^{2} / 2 m$ is the reference energy and $h_{i}$ are adimensional functions (see Appendix $\mathrm{C}$ ).

Inserting these results into Eq. (21) we get

$$
\dot{N}^{>}=-\Gamma_{\text {eq }}\left(r^{>}-r^{<}\right)\left[\left(h_{1}+h_{3}\right) r^{<}+\left(h_{2}+h_{3}\right) r^{>}\right],
$$

or, equivalently, dividing by $N$ :

$$
\dot{x}^{>} \equiv \frac{\dot{N}^{>}}{N}=-\frac{\Gamma_{\mathrm{eq}}}{N}\left(r^{>}-r^{<}\right)\left[\left(h_{1}+h_{3}\right) r^{<}+\left(h_{2}+h_{3}\right) r^{>}\right] .
$$

Therefore, according to this model, the relaxation dynamics of the single-well problem depends only upon the parameter $\beta V_{0}$; all the others $(T, N, \sigma, m$, and the volume $\Omega$ ) enter only through the combination $N / \Gamma_{\text {eq }}$ [see Appendix A, Eq. (A3)] with the result of setting a time scale for the problem, i.e., a prefactor entering the time $\tau$ in which the particles move from above (below) to below (above) the reference energy.

The stationarity condition is

$$
r^{<}=r^{>} \Leftrightarrow N^{>}=N_{\text {eq }}^{>}, \quad N^{<}=N_{\text {eq }}^{<} .
$$

The fraction of particles that at equilibrium is above reference (have $p_{x}^{2}>p_{0}^{2}$ ) can be easily evaluated and is

$$
x_{\mathrm{eq}}^{>} \equiv \frac{N_{\mathrm{eq}}^{>}}{N}=\operatorname{erfc}\left(\sqrt{\beta V_{0}}\right) .
$$

In panel (a) of Fig. 2 we show (lines) the various rates at equilibrium vs $\beta V_{0}$. The rates obtained in the simulation at equilibrium are also shown (points) as a check of the numerical algorithm. The empty symbols denote the rates in a single well, and the full symbols denote the rates in a double well. The presence of the filtering wall prevents collisions between particles on different sides, unless both have high enough energy; we also verified that $\Gamma_{i}$ and $\Gamma_{\text {eq }}$ are separately very similar for the single square well and the SDW since the effect of the tiny barrier on these bulk quantities is negligible.
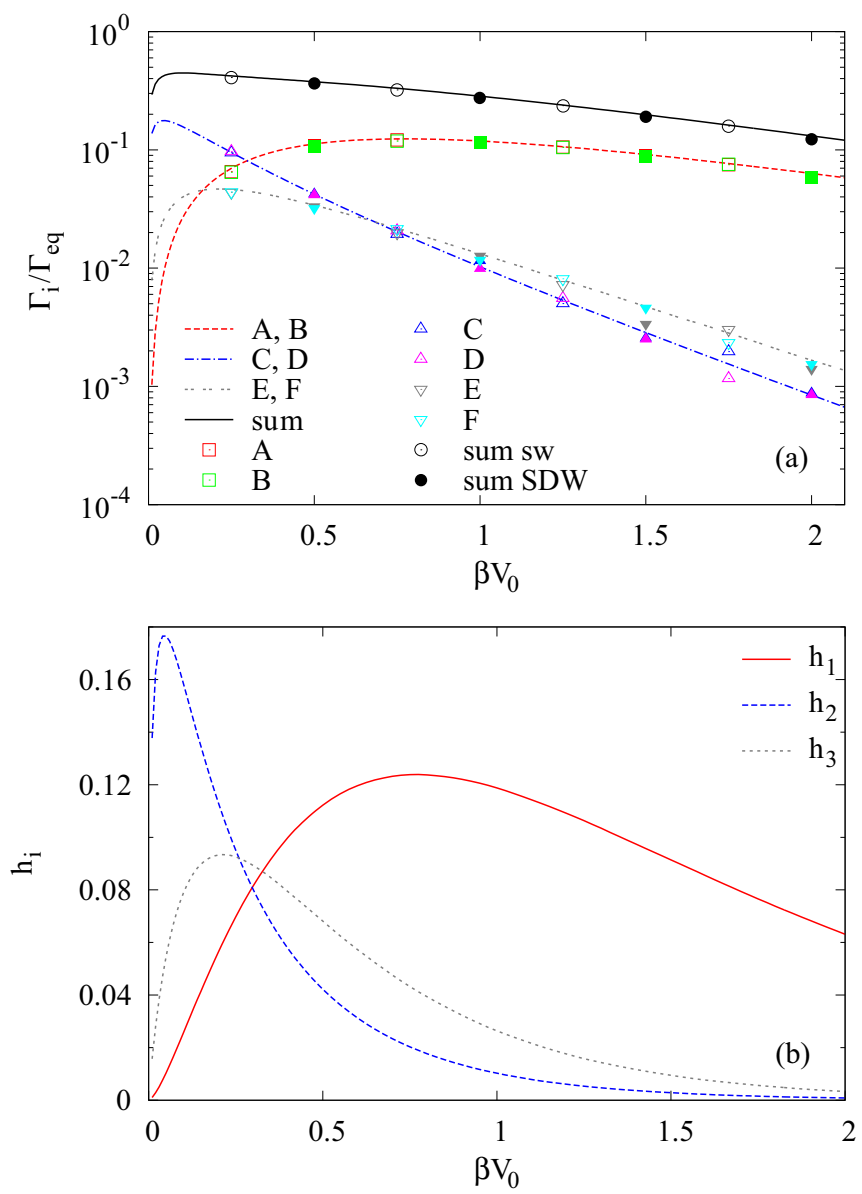

FIG. 2. Rates at equilibrium. Panel (a): equilibrium rates (lines) of the processes $A, B$ (dashed), $C, D$ (dot-dashed), $E$, and $F$ (doubledashed), defined in (20), and their sum vs $\beta V_{0}$. The rates are equal two by two and correspond to $h_{1}, h_{2}, h_{3} / 2$. The simulations (points) were performed for a square single-well potential, with parameters $N=$ 5000, $L=0.5$, and $T=10$. Results with (full symbols) and without (open symbols) a barrier are shown. Panel (b): the adimensional functions $h_{1}, h_{2}$, and $h_{3}$ (with linear scale in vertical axis) vs $\beta V_{0}$.

From panel (a) of Fig. 2 we also see that, out of all the collisions taking place, the processes affecting $N \gtrless$ (full line) are, on the whole, always a percentage smaller than $50 \%$ and, for $\beta V_{0}=2$, have already decreased to $\sim 10 \%$. In our simulations, we will always consider $\beta V_{0}<2$. Concerning the relative importance of the different processes, $A$ and $B$ are the most probable ones for $\beta V_{0} \gtrsim 0.5$.

For completeness, we show in panel (b) the adimensional functions $h_{i}\left(\beta V_{0}\right), i=1,2,3$; notice that these quantities, strictly related to those shown in panel (a) [see Eq. (22) and Eq. (23)], are shown here using a linear scale in the vertical axis.

Finally, we would like to emphasize that, while of course the total collision rate $\Gamma_{\text {eq }}$ depends on the trap shape (see Appendix A), the ratios $\Gamma_{i} / \Gamma_{\text {eq }}$ do not (see Appendix C). In fact, the spatial dependence factors out and cancels between numerator and denominator, leaving only integrals over the momenta. Therefore, also the data obtained in a realistic trap would fall on top of the curves of Fig. 2(a). 
We now discuss the linearization of the dynamical equations (24) and the drawbacks of the analytical model.

\section{Linearization and relaxation time}

The evolution in time of $N^{>}$is ruled by the nonlinear equation (24). If linearized around the equilibrium, it admits an exponentially decaying solution $N^{>}(t)=N_{\text {eq }}^{>}+b e^{-t / \tau}$ with the single-well relaxation time $\tau$ that (omitting details) reads

$$
\tau=\frac{N}{\Gamma_{\mathrm{eq}}} \frac{\operatorname{erfc}\left(\sqrt{\beta V_{0}}\right) \operatorname{erf}\left(\sqrt{\beta V_{0}}\right)}{h_{1}+h_{2}+2 h_{3}}=2 \tau_{\mathrm{coll}} \frac{x_{\mathrm{eq}}^{>}\left(1-x_{\mathrm{eq}}^{>}\right)}{h_{1}+h_{2}+2 h_{3}} .
$$

In the last equality we used Eq. (15) and Eq. (27).

\section{Shortcomings of the analytical model}

The simple assumption Eq. (17) is expressed in terms of just one dynamical degree of freedom, $N^{>}(t)$ : this choice does not allow one to respect energy conservation exactly. The energy of our gas is purely kinetic and can be computed at any time from the distribution function as

$$
E(t)=\int d \Gamma f(\mathbf{p}, t) \frac{p^{2}}{2 m} .
$$

At equilibrium, it yields correctly $E_{\text {eq }}=\frac{3}{2} \frac{N}{\beta}$. However, the assumption Eq. (17) implies the following energy variation:

$$
\frac{\delta E(t)}{E_{\mathrm{eq}}}=\frac{N^{>}(t)-N_{\mathrm{eq}}^{>}}{N} C\left(\sqrt{\beta V_{0}}\right)
$$

where

$$
C(x)=\frac{2}{3 \sqrt{\pi}} \frac{x e^{-x^{2}}}{\operatorname{erfc}(x) \operatorname{erf}(x)} .
$$

The function $C$ is positive for $x \geqslant 0$ : it starts from $C(0)=1 / 3$ and increases for larger values of $x$. So, by construction, energy conservation is violated in the analytical model as soon as $N^{>} \neq N_{\text {eq }}^{>}$. We can expect the model to be better as long as $\left|\delta E(t) / E_{\mathrm{eq}}\right|$ remains small enough during the whole evolution. However, we explored different initial conditions having the same $\Delta N(t=0)$ and we observed that even in cases in which $\left|\delta E(t) / E_{\text {eq }}\right|$ becomes relatively large, the estimate of $\tau$ is good due to a compensation of effects. It also emerged that the estimate of $\tau$ appears to be better when $\delta E$ is positive.

The limitations due to the nonconservation of the energy could be overcome by choosing a structure for $f(\mathbf{p}, t)$ with at least a further independent degree of freedom: for example, one could introduce a variable giving the amount of energy above the reference energy $V_{0}$.

\section{B. Square double well}

We now insert an energy barrier inside the box of Sec. III A, obtaining the SDW potential. To be specific, assume the box is $[-L, L]^{3}$ and the barrier located at $x=0$. The barrier is perfectly transparent for particles with momentum $\left|p_{x}\right|>p_{0}$ and perfectly reflecting for particles with momentum $\left|p_{x}\right|<$ $p_{0}$. The energy associated to the barrier is $V_{0}=p_{0}^{2} / 2 m$.
We can now straightforwardly extend the model seen for the single well to this case: we write

$$
f(\mathbf{p}, t)=f_{L}(\mathbf{p}, t)+f_{R}(\mathbf{p}, t),
$$

as in Eq. (17) with $f_{\alpha}(\mathbf{p}, t)=g_{\alpha}^{>}(\mathbf{p}) N_{\alpha}^{>}(t)+g_{\alpha}^{<}(\mathbf{p}) N_{\alpha}^{<}(t)$ and $\alpha=L, R$ denoting the well. Since the role played by $V_{0}$ is the same as in the single-well model, the $g$ 's depend on $\beta$ and $V_{0}$ in the same way and they do not depend on $\alpha: g_{L}=g_{R} \equiv g$. The numbers of particles in the two wells are $N_{L}=N_{L}^{>}+N_{L}^{<}$and $N_{R}=N_{R}^{>}+N_{R}^{<}$. Now we have four variables or components $N_{L}^{>}, N_{L}^{<}, N_{R}^{>}, N_{R}^{<}$. The equations of motion are

$$
\begin{aligned}
\dot{N}_{L}^{>}= & -\Gamma_{\mathrm{eq}}^{\prime}\left(r_{L}^{>}-r_{L}^{<}\right)\left[\left(h_{1}+h_{3}\right) r_{L}^{<}+\left(h_{2}+h_{3}\right) r_{L}^{>}\right] \\
& -k_{A}\left(N_{L}^{>}-N_{R}^{>}\right), \\
\dot{N}_{L}^{<}= & \Gamma_{\mathrm{eq}}^{\prime}\left(r_{L}^{>}-r_{L}^{<}\right)\left[\left(h_{1}+h_{3}\right) r_{L}^{<}+\left(h_{2}+h_{3}\right) r_{L}^{>}\right], \\
\dot{N}_{R}^{>}= & -\Gamma_{\mathrm{eq}}^{\prime}\left(r_{R}^{>}-r_{R}^{<}\right)\left[\left(h_{1}+h_{3}\right) r_{R}^{<}+\left(h_{2}+h_{3}\right) r_{R}^{>}\right] \\
& +k_{A}\left(N_{L}^{>}-N_{R}^{>}\right), \\
\dot{N}_{R}^{<}= & \Gamma_{\mathrm{eq}}^{\prime}\left(r_{R}^{>}-r_{R}^{<}\right)\left[\left(h_{1}+h_{3}\right) r_{R}^{<}+\left(h_{2}+h_{3}\right) r_{R}^{>}\right] .
\end{aligned}
$$

We have denoted with a ' the quantities referring to $N^{\prime} \equiv N / 2$ particles in a volume $\Omega^{\prime} \equiv \Omega / 2$ : of course, the density is the same and therefore the collision rate per particle is the same too $\left(\Gamma_{\text {eq }}^{\prime} / N^{\prime}=\Gamma_{\text {eq }} / N\right)$.

We observe that $N_{L}^{>}, N_{L}^{<}, N_{R}^{>}, N_{R}^{<}$obey the condition $N_{L}^{>}+$ $N_{L}^{<}+N_{R}^{>}+N_{R}^{<}=N$ ( $N$ is the total number of particles in the double well) resulting in three independent parameters. However, during the first part of the dynamics, the particles above the barrier rapidly flow from one well to the other practically giving $N_{L}^{>} \approx N_{R}^{>}$, and therefore the independent parameters in the subsequent dynamics are in practice just two.

With respect to the single-well case Eq. (24), there is a qualitatively new term coupling the $L$ and $R$ sides of the barrier: it is a diffusion term giving the rate of particles passing from one side of the wall to the other

$$
\left.\dot{N}_{L}^{>}\right|_{\text {diff }}=-\frac{\Delta N_{L \rightarrow R}}{\Delta t}+\frac{\Delta N_{R \rightarrow L}}{\Delta t}=-k_{A}\left(N_{L}^{>}-N_{R}^{>}\right) .
$$

The coefficient $k_{A}$ (Arrhenius) is

$$
k_{A}=\frac{1}{L \sqrt{2 \pi \beta m}} \frac{e^{-\beta V_{0}}}{\operatorname{erfc}\left(\sqrt{\beta V_{0}}\right)} .
$$

A global factor $1 / L$ in $k_{A}$ arises from the ratio between the area of the filtering wall and the volume of each partition: it would be replaced, in a more general geometry, by $\Sigma / \Omega^{\prime}$.

\section{Linearization and relaxation time}

Linearizing Eqs. (33) around equilibrium, we find they admit the following solution:

$$
x_{L}(t)=\frac{1}{2}+c_{1} e^{\lambda_{1} t}+c_{2} e^{\lambda_{2} t}
$$

where the eigenvalues are found to be

$$
\begin{aligned}
\lambda_{1,2} & =-\frac{1}{2}\left[2 k_{A}+k_{s w} \pm \sqrt{\left(2 k_{A}+k_{s w}\right)^{2}-8 k_{s w} k_{A} x_{\mathrm{eq}}^{>}}\right] \\
& \equiv-\frac{1}{\tau_{1,2}} .
\end{aligned}
$$


We have defined the single-well rate $k_{s w} \equiv \frac{1}{\tau_{s w}}$, with $\tau_{s w}$ the single-well relaxation time given by Eq. (28). We also denoted the eigenvalues so that $\tau_{1}$ is larger than $\tau_{2}$. In the comparison with the numerical results (see next sections), since $\tau_{2}$ is associated to the short-time dynamics and $\tau_{1}$ to the long-time one, we plot $\tau_{1}$ as the analytical prediction for the relaxation time. In the limit of large cross sections, $\tau_{1}$ tends to the diffusive limit $\tau_{1, \text { diff }} \equiv \lim _{\sigma \rightarrow \infty} \tau_{1}=L \sqrt{\pi \beta m / 2} e^{\beta V_{0}}$.

Since $x_{\mathrm{eq}}^{>}$is small, one can get more insight approximating the eigenvalues to lowest order in $x_{\mathrm{eq}}^{>}$:

$$
\tau_{1} \simeq\left(\frac{1}{k_{s w}}+\frac{1}{2 k_{A}}\right) \frac{1}{x_{\mathrm{eq}}^{>}}, \quad \tau_{2} \simeq \frac{1}{2 k_{A}+k_{s w}} .
$$

Notice that $\tau_{1}$ is a sum of two terms, one depending on the collision (to which we may refer as $\tau_{1, \text { coll }}$ ) and the other not $\left(\tau_{1, \text { diff }}\right)$. The latter term is dominating for large $\sigma$, and for all $\sigma$ it is $\tau_{1} \geqslant \tau_{1, \text { diff }}$.

We can also write $\tau_{1}$ as

$$
\tau_{1} \simeq L \sqrt{\beta m}\left[\frac{4}{\sqrt{\pi}} \frac{1}{N\left(\frac{d_{\text {int }}}{L}\right)^{2}} \frac{\operatorname{erf}\left(\sqrt{\beta V_{0}}\right)}{h_{1}+h_{2}+2 h_{3}}+\sqrt{\frac{\pi}{2}} e^{\beta V_{0}}\right],
$$

where $d_{\text {int }}=\sqrt{\sigma / \pi}$ is the length scale associated to interactions.

Summarizing, in the cubic box with a filtering barrier (the DSW model) we get

$$
-\frac{1}{\tau_{1}}=-\frac{1}{2}\left[2 k_{A}+k_{s w}-\sqrt{\left(2 k_{A}+k_{s w}\right)^{2}-8 k_{s w} k_{A} x_{\mathrm{eq}}^{>}}\right]
$$

where the coefficients are

$$
\begin{aligned}
k_{s w} & =\frac{\Gamma_{\mathrm{eq}}}{N} \frac{h_{1}+h_{2}+2 h_{3}}{x_{\mathrm{eq}}^{>}\left(1-x_{\mathrm{eq}}^{>}\right)}, \\
k_{A} & =\frac{1}{L \sqrt{2 \pi \beta m}} \frac{e^{-\beta V_{0}}}{x_{\mathrm{eq}}^{>}},
\end{aligned}
$$

and $x_{\text {eq }}^{>}$is given in Eq. (27).

\section{Adapting the analytical model to the harmonic-Gaussian double well}

As an approximation for the realistic double well, we suppose we can still use Eq. (40), but with other coefficients:

$$
\begin{gathered}
k_{s w}^{\mathrm{HGDW}}=\frac{2 \Gamma_{\mathrm{eq}, \mathrm{HO} \text { at min, } N / 2}}{N} \frac{h_{1}+h_{2}+2 h_{3}}{x_{\mathrm{eq}}^{>}\left(1-x_{\mathrm{eq}}^{>}\right)}, \\
k_{A}^{\mathrm{HGDW}}=\frac{\omega_{\min }}{2 \pi} \frac{e^{-\beta V_{0}}}{x_{\mathrm{eq}}^{>}},
\end{gathered}
$$

with $x_{\text {eq }}^{>}$as given in Eq. (27).

In fact, the rates $h_{i}$ are the same in any potential $V(\mathbf{r})$, because they are global quantities in which spatial dependence cancels out (see Appendix C). What changes when passing from the toy to the realistic double well are the rate of collisions, entering $k_{s w}$, and the characteristic frequency, entering $k_{A}$. In Eqs. (43) and (44) we have used the harmonic approximation of the realistic HGDW well close to its minima, where $\omega_{\min }^{2}=2 \ln \left(\tilde{V} / m \omega_{0}^{2} w^{2}\right)$ and $\Gamma_{\text {eq, } \mathrm{HO} \text { at min, } N / 2}$ is the rate for $N / 2$ particles in a harmonic trap of frequencies $\left(\omega_{x}, \omega_{y}, \omega_{z}\right)=\left(\omega_{\min }, \omega_{0}, \omega_{0}\right)$ (see Appendix A). As a consequence of the modification of $k_{A}$, also the expression of the diffusive limit of $\tau_{1}$ is altered into $\tau_{1, \mathrm{diff}}^{\mathrm{HGDW}}=\frac{\pi}{\omega_{\min }} e^{\beta V_{0}}$.

\section{NUMERICAL RESULTS FOR THE SQUARE DOUBLE WELL}

We show in this section the numerical results of test-particle simulations for the SDW model potential. We consider a system with given $N$, density, and temperature $(N=5000$, $L=0.5, T=10$ ) and vary the barrier height $V_{0}$ and the cross section $\sigma$. In all the simulations, the initial population is $60 \%$ in the left well and $40 \%$ in the right one; the initial momentum distribution is the thermal equilibrium one with the same temperature in both wells. A comment is in order about units: in this section we use units in which $\hbar=k_{B}=m=1$ and $2 L=1$.

As an example, we show in Fig. 3 the evolution in time of population imbalance for two values of $V_{0}$. The curves are obtained averaging over 40 runs with different microscopic initial conditions and smoothing over small time intervals. These two procedures are necessary because the barrier crossing is a rare event and leads to large fluctuations in the well population. Notice that this is not a numerical artifact: also in an experimental realization it would be very useful to average over different realizations to be able to observe the population evolution in time clearly for large barriers. As a consequence of the smoothing, the curves in Fig. 3 start from values different from 0.6: for example, in the case of a very low barrier, $V_{0}=1$, the adjustment from $x_{L}=0.6$ to $x_{L} \simeq 0.53$ was very fast.

The obtained behavior can be nicely fitted with a single exponential decay

$$
f_{1}(t)=a+b e^{-t / \tau}
$$

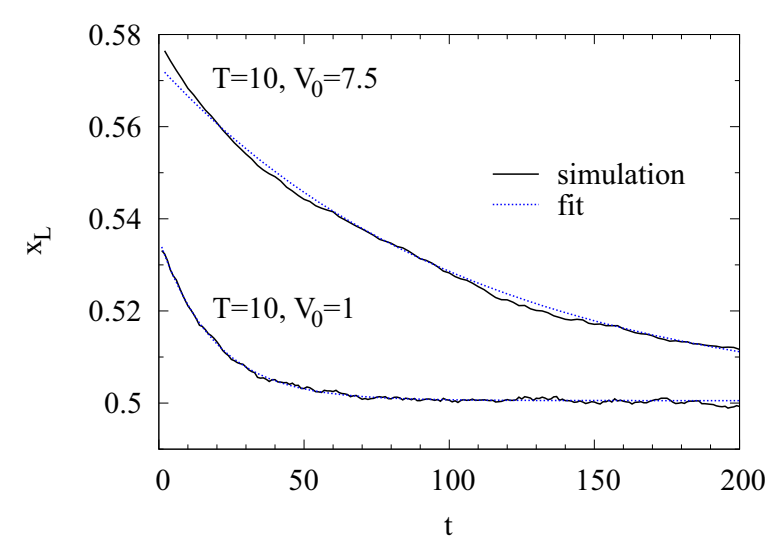

FIG. 3. Dynamics in the SDW potential. Time evolution of the well population for two barrier heights. Parameters: $N=5000, L=$ $0.5, T=10$, and $\sigma / \pi=0.001^{2}$. The barrier height is $V_{0}=1$ for the lower curve and $V_{0}=7.5$ for the upper one. The simulation curves are obtained averaging over 40 runs with different microscopic initial conditions and smoothing over small time intervals. The fits (dotted lines) are done with the function $f_{1}(t)=a+b e^{-t / \tau}$. 


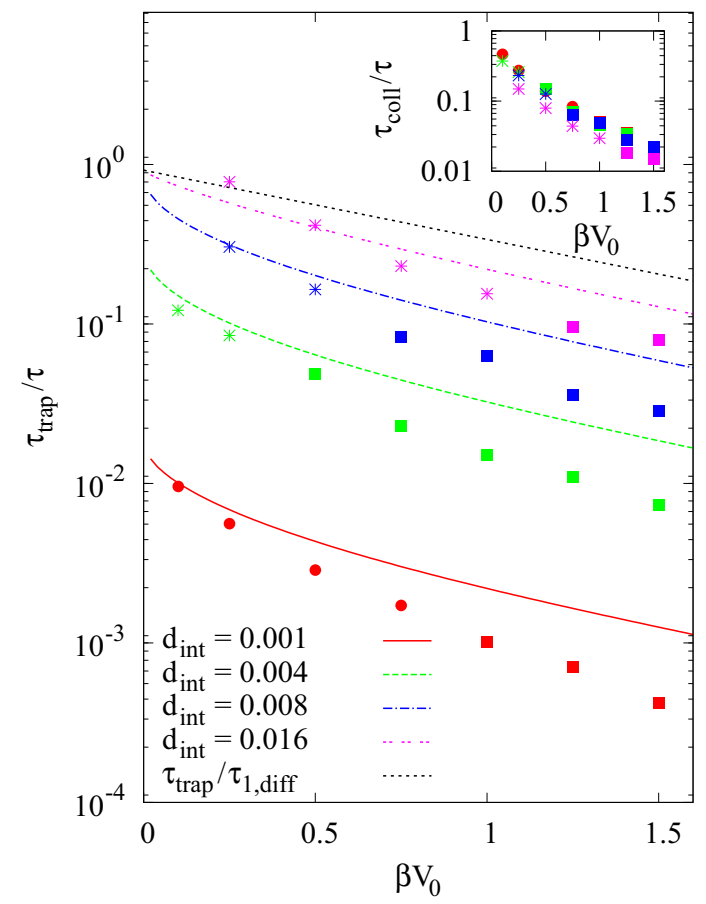

FIG. 4. SDW potential relaxation times. The inverse relaxation time $1 / \tau$, in units of $1 / \tau_{\text {trap }}$, as a function of $\beta V_{0}$ for different values of the cross section: $\sigma=\pi(0.001,0.004,0.008,0.016)^{2}$ with $x_{L}(t=0)=0.6$. The system is a box of size $2 L=1$, with $N=5000$ particles, at $T=10$ and different barriers $V_{0}$. The numerical results are denoted by points. Each point is obtained from an average over 40 runs [64 for the largest value of the cross section $\sigma / \pi=0.016^{2}$ ] and smoothing over small time intervals. The fits are done in the whole time interval, using one of the three functions $f_{1}(t)$ (full circles), $f_{2}(t)$ (stars), and $f_{3}(t)$ (full squares) defined in the text. Different gray scales indicate different values of the cross section. For comparison, we show (lines) the results obtained in an approximated analytical solution presented in Sec. III, namely $\tau_{1}$ obtained using Eqs. (40), (27), (41), and (42). The diffusive limit $\tau_{1, \text { diff }}$ is also shown (short-dashed line). Inset: inverse of $\tau$ rescaled in units of collisional time $\tau_{\text {coll }}$. Gray scales correspond to the same cross section as the main figure.

that allows one to extract the relaxation time $\tau$. For other choices of the cross section or other barrier heights, the evolution in time of $x_{L}(t)$ is sometimes more complex, showing an oscillatory behavior at the early times or an exponential decay with two clearly separated time scales. Therefore, in the following, to extract the relaxation time $\tau$ from $x_{L}(t)$, we will also consider the fitting functions $f_{2}(t)=a+b e^{-t / \tau}+$ $c \cos (\omega t+d) e^{-t / \tau_{2}}$ and $f_{3}(t)=a+b e^{-t / \tau}+c e^{-t / \tau_{2}}$.

In Fig. 4 we show the results for the relaxation time for an initial imbalance of $20 \%$ [ $\left.x_{L}(t=0)=0.6\right]$, and different values of the cross section $\sigma=\pi(0.001,0.004,0.008,0.016)^{2}$ as a function of $\beta V_{0}$. The relaxation time is shown in units of $\tau_{\text {trap }}$, that for the SDW model is $\tau_{\text {trap }}=2 L \sqrt{\beta m / 3}$. Any point is the result of a fit done on a curve obtained averaging 40 (64 in the case of the largest cross section) runs with different microscopic initializations. The fits are done on the whole available time interval, and the chosen fitting function is the one leading to the smaller $\chi^{2}$. The point gray scale indicates the value of the cross section and the point shape the fitting

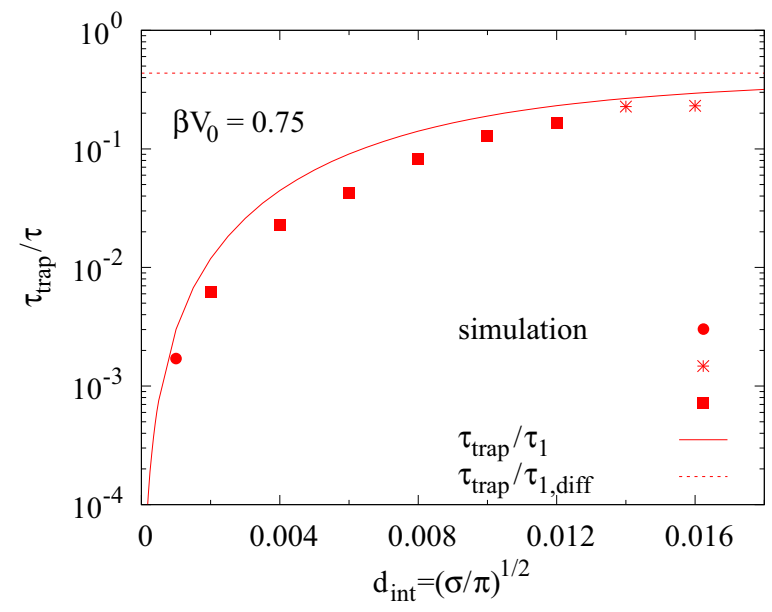

FIG. 5. SDW model potential. Inverse relaxation time $1 / \tau$, in units of $1 / \tau_{\text {trap }}$, as a function of $d_{\text {int }}$ for a given barrier height, $\beta V_{0}=$ 0.75 . Parameters and the meaning of symbols and lines are the same of Fig. 4. The numerical results are denoted by points and the model prediction by lines.

function: $f_{1}$ (full circles), $f_{2}$ (stars), and $f_{3}$ (full squares). In the inset we show again the inverse relaxation time, but in units of the collisional time: in this scale all the points fall in a narrow region (notice that the range of values in the vertical axis of the inset is much smaller than in the main plot), showing that it is the collisional time that gives the major contribution to $\tau$.

In Fig. 5 we focus on a single barrier height and show the dependence of $\tau$ (again in units of $\tau_{\text {trap }}$ ) upon the interaction strength.

\section{Comparison with the analytical model predictions}

In Fig. 4 and Fig. 5 we compare the numerical results with the predictions of the analytical model for the SDW (lines). The model in general predicts a faster relaxation (smaller $\tau$ ) than what was found in the simulation, and as the barrier height increases the agreement is deteriorated. In Fig. 4 one sees, however, that for low and intermediate barriers the agreement is rather satisfactory.

A feature that the model captures nicely is the dependence of the relaxation time upon the interaction strength: in Fig. 4 one can see that, as the cross section increases, both types of curves accumulate towards the diffusive limit. This finding is better seen in Fig. 5, where we fix the relative barrier height at an intermediate value $\left(\beta V_{0}=0.75\right)$ and study the evolution of $\tau$ with the interaction strength.

To better understand the discrepancy between the simulation and the model, and identify a possible improvement of the latter, we have analyzed several moments of the momentum distributions in the simulations (averages of $|\vec{p}|^{i}$ with $i=-2,-1,1,2,3,4)$. In the model we describe the gas with four components $[(<, L),(>, L),(<, R),(>, R)]$ and assume that the population of each of them evolves in time, but the shape of the momentum distribution " $>$ " and " $<$ " is fixed and determined uniquely by $T$ and $V_{0}$ [see Eq. (32)]. Also, we assume that the parameter $T$ is the same for all the four components, and that at any time it is the same it 

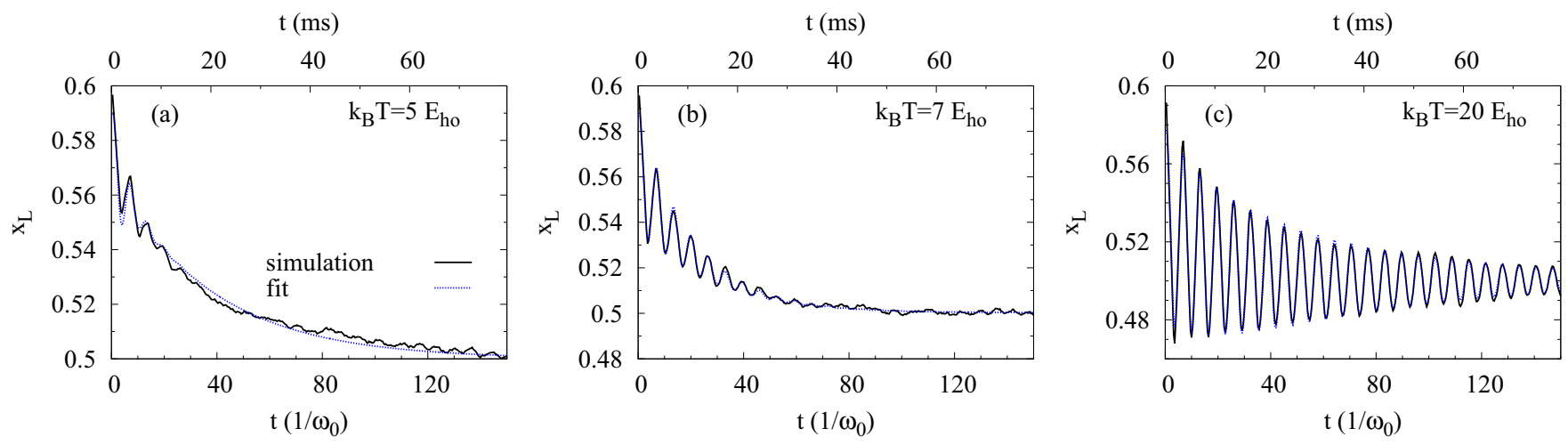

FIG. 6. Dynamics in the HGDW. Evolution of $x_{L}$ with time, for three values of the temperature. The gas contains $N=5000$ particles, with an initial imbalance of $x_{L}(t=0)=0.6$. The well parameters, in trap units, are $\tilde{V}=10, d_{\text {int }}=0.06, w=0.8$ (therefore, $V_{0} \simeq 7.6$ ), and the temperatures $T=5$ [panel (a)], 7 [panel (b)], and 20 [panel (c)]. To express them in dimensional units, reported in the top $x$ axis, we need to specify the trap frequency $\omega_{0}$ and the particle mass: for ${ }^{6} \mathrm{Li}$ atoms and with $\omega_{0}=2 \pi \times 300 \mathrm{~Hz}$ one has $\tilde{V} / k_{B}=144 \mathrm{nK}, d_{\text {int }}=$ $142 \mathrm{~nm}=2684 a_{0}, w=1.89 \mu \mathrm{m}$, and $T=72,101,288 \mathrm{nK}$. These values are realistic for ${ }^{6} \mathrm{Li}$ atoms in a double well potential as in the recent experiment [16] (which is anyway done below $T_{c}$ ). The fits (dotted lines) are done with the function $f_{2}(t)=a+b e^{-t / \tau}+c \cos (\omega t+d) e^{-t / \tau_{2}}$. The values of $\tau$ and $\omega$ fitted from the data for figures (a),(b),(c) in trap units are respectively $\tau=(37.2 \pm 0.2,22.8 \pm 0.2,18.6 \pm 0.4)$ and $\omega=(0.99 \pm 0.03,0.962 \pm 0.002,0.9859 \pm 0.0001)$.

will be at equilibrium. After an analysis of the simulation momentum distributions, we conclude that to improve the analytical model one should promote the temperatures of the " $<$ " components to dynamical degrees of freedom $\left[T_{<, L}(t)\right.$ and $T_{<, R}(t)$ ], and add an extra equation of motion to describe the transport of energy. In fact, during the relaxation, the distributions are compatible with Maxwell-Boltzmann ones, but the temperature $<, L(<, R)$ is smaller (larger) than the equilibrium $T$. In a typical set of simulations the difference between the temperatures is of $\sim \pm 5 \%$ (e.g., for $d=0.008$, $V_{0}=10$, and $T=10$ ). It is not easy to assess the quantitative impact of this difference on the final relaxation time, because the presence of two temperatures in each well would imply a generalization of the formulas for the rates of the various processes [Eq. (22)]. However, we can expect that its effect is twofold, since the temperature affects (1) the total collision rate [see Eq. (A3)] and (2) the relative frequency of a certain process (see Fig. 2). Since, during the equilibration, the left well is more populated, it is reasonable that a lower effective temperature in part of this well will slow down the relaxation.

\section{NUMERICAL RESULTS FOR THE HARMONIC-GAUSSIAN DOUBLE WELL}

In this section we present our results for the realistic HGDW, Eq. (12), that is a combination of a spherical harmonic trap and a Gaussian barrier along one direction. We start with an initial configuration with $60 \%$ of the particles in the left well and $40 \%$ in the right one; their initial momentum distribution is the thermal equilibrium one with the same temperature in both wells. To prepare this configuration numerically, we start with the balanced population and then move $10 \%$ of the particles from the right to the left well.

We then let the system evolve: $x_{L}(t)$ results from a combination of a center-of-mass oscillation due to the harmonic trapping, and the relaxation of population imbalance. We choose to fit such behavior with the $f_{2}$ fit function $f_{2}(t)=a+$ $b e^{-t / \tau}+c \cos (\omega t+d) e^{-t / \tau_{2}}$, where the third term represents the damped center-of-mass oscillation. We have checked this interpretation exciting explicitly the c.o.m. motion, i.e., considering a balanced cloud and displacing it as a whole by a certain amount. The frequency and damping of this c.o.m. mode are in reasonable agreement with those extracted from the oscillation that arises when the system is prepared with an initial population imbalance and not displaced.

We fix the double-well parameters and study the relaxation dynamics for different temperatures and interactions. In all the cases we have $N=5000$ particles and the initial imbalance is $20 \%$ [i.e., $x_{L}(t=0)=0.6$ ]. The curves are obtained averaging over 40 different microscopic realizations and smoothing over small time intervals. Since we are in the presence of a harmonic trap, in this section we use the harmonic-oscillator units (or trap units), in which all the dimensional quantities are built from $\hbar, k_{B}, m, \omega_{0}$ as usual. For example, $E_{h o}=\hbar \omega_{0}, l_{h o}=\sqrt{\hbar / m \omega_{0}}$, and so on. To pass to physical units, values have to be chosen for the mass $m$ and the trap frequency $\omega_{0}$. The well parameters we use, in trap units, are $\tilde{V}=10$ and $w=0.8$ (therefore, $V_{0} \simeq 7.6$ ).

In Fig. 6 we show three typical behaviors of $x_{L}(t)$ : they correspond to a given cross section and different temperatures, increasing from left to right. The frequency $\omega$ of the c.o.m. oscillation is very close to $\omega_{0}$ at high temperatures, where the presence of the barrier does almost not affect the cloud oscillation; at lower temperatures, instead, it is reduced to smaller values.

Repeating similar calculations for a set of temperatures and interaction strengths, we obtain the results shown in Fig. 7. In some cases, in which the c.o.m. oscillation is not visible anymore, we use the fitting function $f_{3}$ to extract the relaxation time. As before, the symbol shape indicates the fitting function used, with a notation similar to that of Fig. 4. Comparing with the analogous plot for the SDW, Fig. 4, we see an analogous accumulation of curves as the cross section increases. The dependence upon $\beta V_{0}$ seems qualitatively different: the point is that here, along a curve for a given cross section, the global collision rate changes, whereas this was not the case in Fig. 4. 


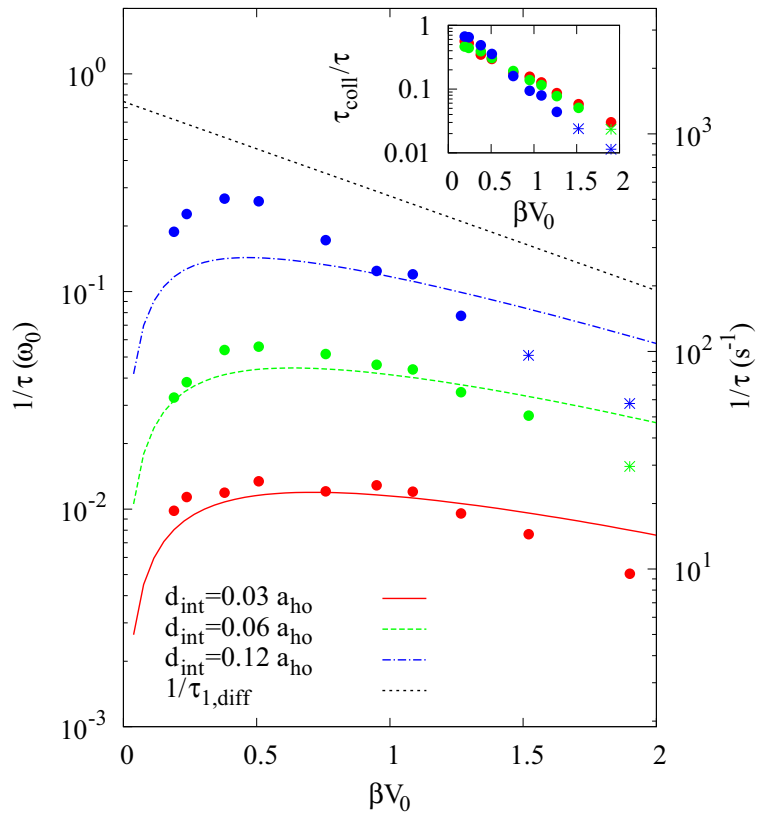

FIG. 7. HGDW relaxation time. The inverse relaxation time $1 / \tau$ as a function of $\beta V_{0}$, obtained varying the temperature (the trap is fixed as in Fig. 6 with a barrier height $V_{0} \simeq 7.6$ ). The numerical results are denoted by points. Different gray scales correspond to different values of the cross section: $\sigma=\pi(0.03,0.06,0.12)^{2}=$ $\pi(71,142,284)^{2} \mathrm{~nm}^{2}$. Each point is obtained from an average over 40 runs. The fits are done in the whole time interval, using either $f_{2}(t)$ (full circles) or $f_{3}(t)$ (stars). The dimensional quantities reported in the figure are obtained as in Fig. 6. For comparison, we show as lines the results obtained in an approximated analytical solution presented in Sec. III, namely $\tau_{1}$ obtained using Eqs. (40), (27), (43), and (44). Inset: inverse of $\tau$ rescaled in units of collisional time $\tau_{\text {coll }}$. Gray scales correspond to the same cross section as the main figure.

If we rescale all the curves by the corresponding equilibrium collision rate (as shown in the inset of the Fig. 7), we see that they all fall in the same region and the trend so obtained is similar in the SDW and in the HGDW.

In Fig. 7, together with the numerical results, we show also (lines) the analytical predictions obtained extending our model to the realistic double-well case (see Sec. III C). Next, in Fig. 8, we show the analogous of Fig. 5 for the realistic HGDW well, for $\beta V_{0} \simeq 0.76$ : the qualitative behavior is the same and it is nicely reproduced by the analytical model.

In the figures of this section (Figs. 6-8), together with the harmonic-oscillator units, we show also axes with physical units: they are obtained assuming the ${ }^{6} \mathrm{Li}$ mass and a trap frequency $\omega_{0}=2 \pi \times 300 \mathrm{~Hz}$. With the considered dimensionless values given above, one obtains realistic values for ${ }^{6} \mathrm{Li}$ atoms in a double-well potential as in the recent experiment [16]: the main difference between the parameter values used here and the experimental ones in [16] is that the experiment reported in [16] is done for superfluid fermions below $T_{c}$, while here we are exploring temperatures above $T_{c}$.

\section{Estimates for a two-component Fermi gas}

Finally, we can use our results to make an approximate prediction for a balanced two-component mixture of ${ }^{6} \mathrm{Li}$.

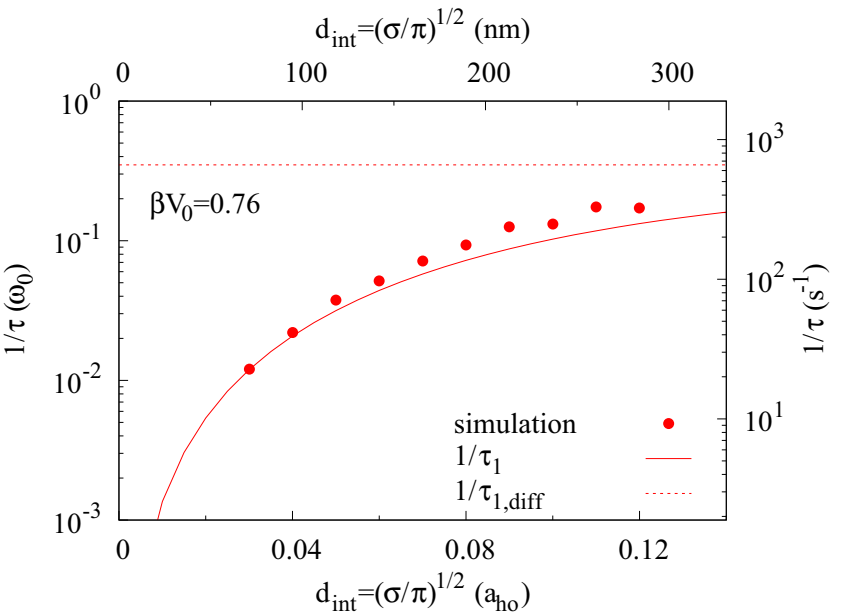

FIG. 8. HGDW potential. Inverse relaxation time $1 / \tau$ as a function of $d_{\text {int }}$ for the same trap of Fig. 6 and a given temperature ( $T=10$, then $\beta V_{0}=0.76$ ). The numerical results are denoted by points and the model prediction by lines. The meaning of symbols and lines are the same of Fig. 7. The dimensional units are obtained as explained in the caption of Fig. 6.

In fact, in this case we would have two species, equally populated $\left(N_{\uparrow}=N_{\downarrow}\right)$ with only interspecies interactions. In the Boltzmann framework, we would have two distribution functions, but they coincide if the mode and the potential do not depend upon the "spin": just one distribution normalized to $N_{\uparrow}$ is needed. Our calculations for 5000 classical particles correspond therefore to a balanced mixture of $10^{4}$ fermions. Anyway, notice that we are not including Pauli blocking in the collision term and we are approximating the cross section with a constant. Of course, the one we present is not a full calculation for fermions. However, we recall that in the context of collective modes it has been found that the inclusion of Pauli blocking alone may lead to results that are in worse agreement with experiment than classical results. In a refined theory one should include both Pauli blocking and medium effects, that turn out to compensate each other in particular for large interactions and this cancellation leads to a remarkable agreement with classical Boltzmann results above $T_{c}$ as discussed for unitary fermions [25,30]. Therefore, we expect that results based on the classical Boltzmann equation without inclusion of in-medium effects and Pauli blocking may give reasonable estimates for our double-well potential, especially for small initial imbalances.

With this in mind, in Fig. 9 we plot the same results of Fig. 7 , as a function of $T / T_{F}$, with $T_{F}=\left(6 N_{\uparrow}\right)^{1 / 3}$ the Fermi temperature in a harmonic trap, and $N_{\uparrow}=5000$. We observe that in the thermal regime higher-order partial waves can play a role [22]: however, we estimated that such effects are important for temperatures much larger than $T_{F}$, while our simulations are between $\sim 0.1 T_{F}$ and $\sim 1.3 T_{F}$.

Notice that, even if we are not explicitly considering the effect of the quantum statistics on the collision term, both numerical computations and the analytical model can be straightforwardly extended to include it: we expect that, at temperatures $T / T_{F} \gtrsim 0.5$ (and in any case larger than $T_{c}$ ), the relaxation time has a similar qualitative dependence on barrier heights and interaction strengths. 


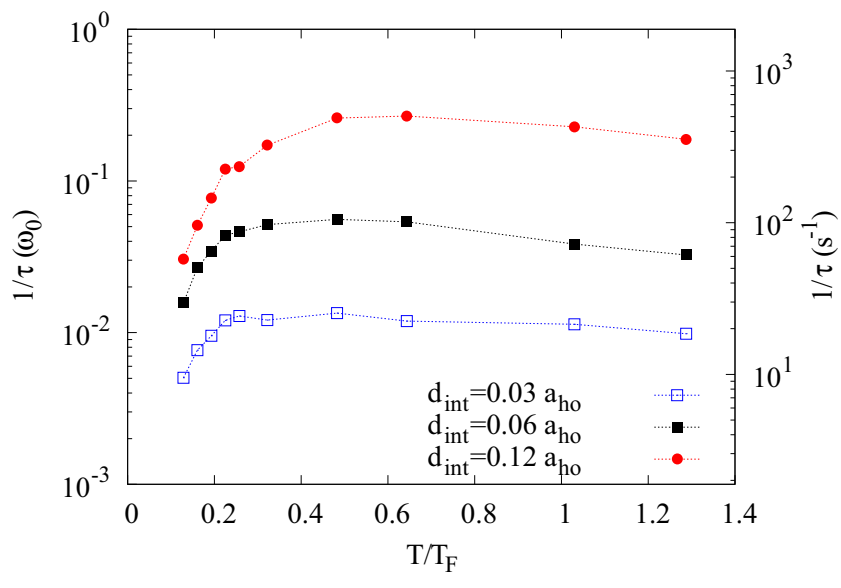

FIG. 9. HGDW potential. Inverse relaxation time $1 / \tau$ as a function of $T / T_{F}$, for a fixed barrier $V_{0} \simeq 7.6$. The results for $\tau$ are the same of Fig. 7, here shown in a different representation. The Fermi temperature has been computed as $T_{F}=\left(6 N_{\uparrow}\right)^{1 / 3}$ : that is, the Fermi temperature for a balanced mixture having $N_{\uparrow}=N_{\downarrow}=N$ in a harmonic well. For details on the parameters and the dimensional units, see the caption of Fig. 6.

\section{CONCLUSIONS}

In this work we presented a study of the double-well dynamics of a classical gas that obeys the Boltzmann equation, with the purpose of assessing the role played by collisions in the relaxation of population imbalance between the two wells. We think that a detailed study of the Boltzmann equation in a variety of double-well potentials is a rewarding subject of interest, not only for its paradigmatic and pedagogical importance, but also to concretely model currently ongoing experiments in a range of temperature $T \gtrsim T_{c}\left(T_{c}\right.$ being the critical temperature for superfluidity) and to set a basis for further quantitative theoretical studies of tunneling of ultracold atoms at finite temperature below $T_{c}$, in particular near $T_{c}$.

Two model potentials have been considered: a toy square double-well (SDW) potential with a filtering wall and a more realistic double well obtained by combining a Gaussian to a harmonic potential (HGDW) which is of interest for experimental realizations with ultracold atoms. In both cases, we have performed numerical simulations (test-particle method) of the relaxation dynamics from an initial imbalanced population of the symmetric wells to the balanced equilibrium, in a range of cross sections $\sigma$ values and barrier heights $V_{0} / T$. For convenience, in the toy SDW potential we have fixed the temperature and varied the barrier height, whereas in the realistic HGDW one we have fixed the trap shape, therefore the barrier height, and varied the temperature. We have also provided a detailed discussion of the time scales of the problem, namely the collisional time $\tau_{\text {coll }}$, the time associated to the external potential $\tau_{\text {trap }}$, and the time $\tau$ with which the population imbalance equilibrates (see Sec. II D).

Our numerical results obtained using the test-particle method show that the particles already above the barrier at the initial time spread fast across the system. The main contribution to the relaxation time $\tau$ comes from the particles below barrier which can reach the other well through scattering processes. We find that $\tau$ exponentially increases with the barrier height, as expected, and that the larger is the interaction the faster is the relaxation. Our numerical results indicate that, for $\beta V_{0}$ fixed, when increasing the interaction strength the relaxation time saturates to a finite value.

Beside the numerical results, we have also presented an analytical model for the dynamics that allows one to compute the relaxation time analytically from the system parameters. The dynamical variables of our model are the number of particles in each well, below and above the potential barrier, which satisfy a set of nonlinear differential equations in which parameters depending upon the temperature and the double-well pontential enter. Upon linearization, our simple model predicts a fast relaxation time $\tau_{2}$ and a slow relaxation time $\tau_{1}$ which, in the limit of large cross sections, tends to the diffusive limit $\tau_{1} \sim e^{\beta V_{0}}$, also in agreement with an Arrhenius-type law. An advantage of the model is that it also yields an estimate of the prefactor in front of $e^{\beta V_{0}}$ in $\tau_{1}$. The model also predicts that the relaxation time saturates to a finite value for increasing cross sections.

The physical content of our work can be summarized by observing that the thermal Boltzmann dynamics of a classical gas in a double-well potential can be efficiently simulated through the test-particle method. The results can be interpreted with a simple analytical model which shows a reasonable agreement with the numerical findings for low up to intermediate barriers $\left(\beta V_{0} \lesssim 1\right)$. The agreement improves in the case of the realistic HGDW potential in a wide range of barrier heights and interaction cross sections.

The relaxation of some of the constraints we imposed on the distribution functions in the analytical model may naturally provide a better agreement with the numerics. Monitoring the momentum distributions of the simulation allowed us to identify and discuss possible improvements of the analytical model. In particular a concrete possibility would be to put time-dependent effective temperatures below the barrier.

As a possible continuation of this study, it would be interesting to compare our results with those obtained in the framework of a Klein-Kramers equation in the presence of a barrier [1]: to this end, the friction parameter appearing in the Klein-Kramers equation should be appropriately (we mean, quantitatively) computed.

In the final part we used our results to estimate the relaxation times for a set of parameters which are realistic for ongoing experiments with mixtures of fermionic ${ }^{6} \mathrm{Li}$ cold atoms in a double-well potential above the critical temperature. Another extension of this work, relevant for ultracold atom experiments at low temperature, would be to include, beyond the classical crossing mechanism studied here, the hopping via quantum tunneling: a possible way could be to include an effective $\mathrm{ad}$ hoc term in the Boltzmann equation or to couple the Boltzmann equation to the equations for the superfluid dynamics.

\section{ACKNOWLEDGMENTS}

S.C. is supported by the "Fundação para a Ciência e a Tecnologia" (FCT, Portugal) and the "European Social Fund" (ESF) via postdoctoral Grant No. SFRH/BPD/64405/2009. A.T. acknowledges support from the Italian PRIN "Fenomeni quantistici collettivi: dai sistemi fortemente correlati ai simu- 
latori quantistici” (PRIN 2010_2010LLKJBX). This work has been supported by the project MatterWave acknowledging the financial support of the Future and Emerging Technologies (FET) programme within the 7th Framework Programme for Research of the European Commission, under FET Grant No. FP7-ICT-601180. The authors are grateful to D. Davesne, A. Gambassi, A. Laio, and I. Vidaña for valuable discussions. We also gratefully thank for many useful discussions people of LENS and QSTAR groups in Florence, in particular A. Burchianti, K. Xhani, G. Roati, A. Smerzi, and M. Zaccanti. A.T. acknowledges the University of Coimbra for kind hospitality and the Isaac Newton Institute for Mathematical Sciences, Programme "Mathematical Aspects of Quantum Integrable Models in and out of Equilibrium", where the final part of this work was completed. S.C. and T.M. acknowledge CNR-IOM and SISSA for kind hospitality in Trieste during the completion of the work. T.M. acknowledges CNPq for support through the fellowship Bolsa de produtividade em Pesquisa No. 311079/2015-6. The authors also thank the Laboratory for Advanced Computing at the University of Coimbra for providing CPU time in the Milipeia and Navigator clusters.

\section{APPENDIX A: COLLISION RATES AT EQUILIBRIUM}

In a generic potential and for constant cross section, the collision rate at equilibrium reads

$$
\Gamma_{\mathrm{eq}}=\frac{2 \sigma}{\sqrt{\pi \beta m}} \int d^{3} r \rho_{\mathrm{eq}}^{2}(\mathbf{r}),
$$

where $\rho_{\mathrm{eq}}(\mathbf{r})=\int d^{3} p /(2 \pi \hbar)^{3} f_{\mathrm{eq}}(\mathbf{r}, \mathbf{p})$ is the local equilibrium density: in some cases, this integral can be performed analytically.

For a box with hard walls, the distribution function reads

$$
f_{\text {eq }}(\mathbf{p})=e^{-\beta\left(\frac{p^{2}}{2 m}-\mu\right)},
$$

where $\mu$ the chemical potential. The normalization condition $\int d \Gamma f=N$ sets the value of the chemical potential $e^{\beta \mu}=$ $\hbar^{3}(2 \pi \beta / m)^{3 / 2} \rho$ where $\rho=N / \Omega$. One then finds

$$
\Gamma_{\mathrm{eq}}^{\mathrm{box}}=\frac{2 N^{2} \sigma}{\Omega \sqrt{\pi \beta m}}=\frac{2 N \rho \sigma}{\sqrt{\pi \beta m}} .
$$

The average time in a square single well between two consecutive collisions of the same particle is

$$
\tau_{\text {coll }}^{\text {box }}=\left(2 \Gamma_{\mathrm{eq}}^{\mathrm{box}} / N\right)^{-1}=\frac{1}{4} \frac{\sqrt{\pi \beta m}}{\rho \sigma} .
$$

On the other hand, the average time a particle needs to travel across the whole box is

$$
\tau_{\text {trap }}^{\text {box }}=\frac{\Omega^{1 / 3}}{v_{\text {rms }}}=\Omega^{1 / 3} \sqrt{\frac{\beta m}{3}},
$$

where the root-mean-square velocity at equilibrium is $v_{\mathrm{rms}}=$ $\sqrt{3 /(\beta m)}$. Defining the adimensional quantity $\alpha \equiv \tau_{\text {coll }}^{\text {box }} / \tau_{\text {trap }}^{\text {box }}$, if $\alpha \rightarrow 0$ the gas is hydrodynamical, whereas it is collisionless if $\alpha \rightarrow \infty$. The condition on $\alpha$ turns out to be a purely geometrical one since

$$
\alpha=\frac{\sqrt{3 \pi}}{4} \frac{1}{\Omega^{1 / 3} \rho \sigma}:
$$

if the volume and number of particles are fixed, it is a condition on the cross section $\sigma$.

Finally, we use in the text the equilibrium collision rate for a harmonic anisotropic trap $V(\mathbf{r})=m\left(\omega_{x}^{2} x^{2}+\omega_{y}^{2} y^{2}+\right.$ $\left.\omega_{z}^{2} z^{2}\right) / 2$, which is given by

$$
\Gamma_{\mathrm{eq}}^{\mathrm{HO}}=\frac{\sigma N^{2} \beta m \omega_{x} \omega_{y} \omega_{z}}{4 \pi^{2}} .
$$

\section{APPENDIX B: CHEMICAL POTENTIALS FOR THE SQUARE SINGLE WELL}

Regarding the chemical potential, in a square single well $\mu$ is such that

$$
e^{-\beta \mu}=\frac{1}{N} \frac{\Omega}{(2 \pi \beta / m)^{3 / 2} \hbar^{3}} .
$$

On the other hand, the chemical potentials $\mu \gtrless$ entering the analytical model presented in Sec. III are given by

$$
e^{-\beta \mu^{<}}=\frac{\Omega}{(2 \pi \beta / m)^{3 / 2} \hbar^{3}} \operatorname{erf}\left(\sqrt{\beta V_{0}}\right)
$$

and

$$
e^{-\beta \mu^{>}}=\frac{\Omega}{(2 \pi \beta / m)^{3 / 2} \hbar^{3}} \operatorname{erfc}\left(\sqrt{\beta V_{0}}\right) .
$$

The equilibrium values of $N \gtrless$ are

$$
N_{\text {eq }}^{>}=N \operatorname{erfc}\left(\sqrt{\beta V_{0}}\right), \quad N_{\text {eq }}^{<}=N \operatorname{erf}\left(\sqrt{\beta V_{0}}\right) .
$$

\section{APPENDIX C: RATE COEFFICIENTS $\gamma_{i}$ AND $\boldsymbol{h}_{i}$}

For a gas of $N$ particles in a volume $\Omega$, the rates introduced in Sec. III A [see relations (20), (21), and (22)] are

$$
\begin{aligned}
& \gamma_{1}=\Gamma_{A}^{\mathrm{eq}}=e^{2 \beta \mu} \Omega \int \frac{d^{3} p_{1} d^{3} p_{2}}{(2 \pi \hbar)^{6}} \frac{d \sigma}{d \Omega^{\prime}} d \Omega^{\prime} \frac{\left|\mathbf{p}_{1}-\mathbf{p}_{2}\right|}{m} e^{-\frac{p_{1}^{2}+p_{2}^{2}}{2 m}} \Theta\left(p_{1 x}^{2}-p_{0}^{2}\right) \Theta\left(p_{0}^{2}-p_{2 x}^{2}\right) \Theta\left(p_{0}^{2}-p_{1 x}^{\prime 2}\right) \Theta\left(p_{0}^{2}-p_{2 x}^{\prime 2}\right), \\
& \gamma_{2}=\Gamma_{C}^{\mathrm{eq}}=e^{2 \beta \mu} \Omega \int \frac{d^{3} p_{1} d^{3} p_{2}}{(2 \pi \hbar)^{6}} \frac{d \sigma}{d \Omega^{\prime}} d \Omega^{\prime} \frac{\left|\mathbf{p}_{1}-\mathbf{p}_{2}\right|}{m} e^{-\frac{p_{1}^{2}+p_{2}^{2}}{2 m}} \Theta\left(p_{0}^{2}-p_{1 x}^{2}\right) \Theta\left(p_{2 x}^{2}-p_{0}^{2}\right) \Theta\left(p_{1 x}^{\prime 2}-p_{0}^{2}\right) \Theta\left(p^{\prime 2}{ }_{2 x}-p_{0}^{2}\right), \\
& \gamma_{3}=2 \Gamma_{E}^{\mathrm{eq}}=e^{2 \beta \mu} \Omega \int \frac{d^{3} p_{1} d^{3} p_{2}}{(2 \pi \hbar)^{6}} \frac{d \sigma}{d \Omega^{\prime}} d \Omega^{\prime} \frac{\left|\mathbf{p}_{1}-\mathbf{p}_{2}\right|}{m} e^{-\frac{p_{1}^{2}+p_{2}^{2}}{2 m}} \Theta\left(p_{0}^{2}-p_{1 x}^{2}\right) \Theta\left(p_{0}^{2}-p_{2 x}^{2}\right) \Theta\left(p^{\prime 2}{ }_{1 x}-p_{0}^{2}\right) \Theta\left(p^{\prime 2}{ }_{2 x}-p_{0}^{2}\right),
\end{aligned}
$$


where the prefactors come from the relation $e^{\beta \mu_{>}} N_{\mathrm{eq}}^{>}=e^{\beta \mu_{<}} N_{\mathrm{eq}}^{<}=e^{\beta \mu}$ that can be easily checked. The equilibrium total collision rate is

$$
\Gamma_{\mathrm{eq}}=\frac{1}{2} e^{2 \beta \mu} \Omega \int \frac{d^{3} p_{1} d^{3} p_{2}}{(2 \pi \hbar)^{6}} \frac{d \sigma}{d \Omega^{\prime}} d \Omega^{\prime} \frac{\left|\mathbf{p}_{1}-\mathbf{p}_{2}\right|}{m} e^{-\frac{p_{1}^{2}+p_{2}^{2}}{2 m}} .
$$

By passing to adimensional variables, it's easy to see that $h_{i} \equiv \gamma_{i} / \Gamma_{\text {eq }}$ are functions of $\beta p_{0}^{2} / 2 m$ (i.e., of $\beta V_{0}$ ) only.

Notice that these ratios are global equilibrium quantities. We computed them in the uniform case; however, they are the same in any potential $V(\mathbf{r})$ : in fact, the spatial dependence would factorize out and cancel between numerator and denominator.

[1] P. Hanggi, P. Talkner, and M. Borkovec, Rev. Mod. Phys. 62, 251 (1990).

[2] D. F. Calef and J. M. Deutsch, Annu. Rev. Phys. Chem. 34, 493 (1983).

[3] A. Barone and G. Paternó, Physics and Applications of the Josephson Effect (John Wiley and Sons, New York, 1982).

[4] R. Rajaraman, Solitons and Instantons (North-Holland, Amsterdam, 1987).

[5] M. Razavy, Quantum Theory of Tunneling (World Scientific, Singapore, 2003).

[6] D. R. Tilley and J. Tilley, Superfluidity and Superconductivity (Adam Hilger, Bristol, 1990).

[7] J. F. Annett, Superconductivity, Superfluids, and Condensates (Oxford University Press, Oxford, 2004).

[8] T. Macrì and A. Trombettoni, Laser Phys. 23, 095501 (2013).

[9] M. Lewenstein, A. Sanpera, and V. Ahufinger, Ultracold Atoms in Optical Lattices: Simulating Quantum Many-body Systems (Oxford University Press, Oxford, 2012).

[10] A. Smerzi, S. Fantoni, S. Giovanazzi, and S. R. Shenoy, Phys. Rev. Lett. 79, 4950 (1997).

[11] F. S. Cataliotti, S. Burger, C. Fort, P. Maddaloni, F. Minardi, A. Trombettoni, A. Smerzi, and M. Inguscio, Science 293, 843 (2001).

[12] M. Albiez, R. Gati, J. Folling, S. Hunsmann, M. Cristiani, and M. K. Oberthaler, Phys. Rev. Lett. 95, 010402 (2005).

[13] T. Anker, M. Albiez, R. Gati, S. Hunsmann, B. Eiermann, A. Trombettoni, and M. K. Oberthaler, Phys. Rev. Lett. 94, 020403 (2005).

[14] S. Levy, E. Lahoud, I. Shomroni, and J. Steinhauer, Nature 449, 579 (2007).

[15] The BCS-BEC Crossover and Unitary Fermi Gas, edited by W. Zwerger (Springer, Heidelberg, 2012).

[16] G. Valtolina, A. Burchianti, A. Amico, E. Neri, K. Xhani, J. A. Seman, A. Trombettoni, A. Smerzi, M. Zaccanti, M. Inguscio, and G. Roati, Science 350, 1505 (2015).

[17] E. M. Lifshitz and L. P. Pitaevskii, Physical Kinetics, Course of Theoretical Physics Vol. 10 (Pergamon, London, 1981).

[18] C. J. Pethick and H. Smith, Bose-Einstein Condensation in Dilute Gases, 2nd ed. (Cambridge University Press, Cambridge, England, 2008), Chap. 11.

[19] D. Guéry-Odelin, F. Zambelli, J. Dalibard, and S. Stringari, Phys. Rev. A 60, 4851 (1999).

[20] P. Pedri, D. Guéry-Odelin, and S. Stringari, Phys. Rev. A 68, 043608 (2003).

[21] P. Massignan, G. M. Bruun, and H. Smith, Phys. Rev. A 71, 033607 (2005).
[22] M. Anderlini and D. Guéry-Odelin, Phys. Rev. A 73, 032706 (2006).

[23] G. M. Bruun and H. Smith, Phys. Rev. A 76, 045602 (2007).

[24] M. Urban, Phys. Rev. A 78, 053619 (2008).

[25] S. Chiacchiera, T. Lepers, D. Davesne, and M. Urban, Phys. Rev. A 79, 033613 (2009).

[26] O. Goulko, F. Chevy, and C. Lobo, Phys. Rev. A 84, 051605(R) (2011).

[27] D. S. Lobser, A. E. S. Barentine, E. A. Cornell, and H. J. Lewandowski, Nat. Phys. 11, 1009 (2015).

[28] D. Guery-Odelin, J. G. Muga, M. J. Ruiz-Montero, and E. Trizac, Phys. Rev. Lett. 112, 180602 (2014).

[29] H. Haug and A.-P. Jauho, Quantum Kinetics in Transport and Optics of Semiconductors (Springer, Berlin, 2008).

[30] S. Riedl, E. R. Sánchez Guajardo, C. Kohstall, A. Altmeyer, M. J. Wright, J. H. Denschlag, R. Grimm, G. M. Bruun, and H. Smith, Phys. Rev. A 78, 053609 (2008).

[31] S. Chiacchiera, T. Lepers, D. Davesne, and M. Urban, Phys. Rev. A 84, 043634 (2011).

[32] P.-A. Pantel, D. Davesne, S. Chiacchiera, and M. Urban, Phys. Rev. A 86, 023635 (2012).

[33] P.-A. Pantel, D. Davesne, and M. Urban, Phys. Rev. A 91, 013627 (2015).

[34] A. Griffin, T. Nikuni, and E. Zaremba, Bose-condensed Gases at Finite Temperatures (Cambridge University Press, Cambridge, UK, 2009).

[35] M. Urban, Phys. Rev. A 75, 053607 (2007).

[36] K. Iigaya, S. Konabe, I. Danshita, and T. Nikuni, Phys. Rev. A 74, 053611 (2006).

[37] S. Konabe and T. Nikuni, J. Low Temp. Phys. 150, 12 (2008).

[38] D. McKay, M. White, M. Pasienski, and B. DeMarco, Nature (London) 453, 76 (2008).

[39] V. I. Yukalov and E. P. Yukalova, Phys. Rev. A 78, 063610 (2008).

[40] U. Schneider, L. Hackermuller, J. P. Ronzheimer, S. Will, S. Braun, Th. Best, I. Bloch, E. Demler, S. Mandt, D. Rasch, and A. Rosch, Nat. Phys. 8, 213 (2012).

[41] G. Orso, L. P. Pitaevskii, and S. Stringari, Phys. Rev. Lett. 93, 020404 (2004).

[42] L. Pezzè, L. Pitaevskii, A. Smerzi, S. Stringari, G. Modugno, E. De Mirandes, F. Ferlaino, H. Ott, G. Roati, and M. Inguscio, Phys. Rev. Lett. 93, 120401 (2004).

[43] G. F. Bertsch and S. Das Gupta, Phys. Rep. 160, 189 (1988).

[44] B. Jackson and E. Zaremba, Phys. Rev. A 66, 033606 (2002).

[45] F. Toschi, P. Vignolo, S. Succi, and M. P. Tosi, Phys. Rev. A 67, 041605(R) (2003). 
[46] T. Lepers, D. Davesne, S. Chiacchiera, and M. Urban, Phys. Rev. A 82, 023609 (2010).

[47] A. C. J. Wade, D. Baillie, and P. B. Blakie, Phys. Rev. A 84, 023612 (2011).

[48] M. P. Allen and D. J. Tildesley, Computer Simulation of Liquids (Claredon Press, Oxford, 1987).

[49] D. Jaksch, C. Bruder, J. I. Cirac, C. W. Gardiner, and P. Zoller, Phys. Rev. Lett. 81, 3108 (1998).
[50] A. Trombettoni and A. Smerzi, Phys. Rev. Lett. 86, 2353 (2001).

[51] M. J. DeWeert and S. M. Girvin, Phys. Rev. B 37, 3428 (1988).

[52] S. Paganelli and S. Ciuchi, J. Phys.: Condens. Matter 20, 235203 (2008).

[53] More precisely, in the presence of a barrier in a certain direction, say $x$, the particles are divided in two classes: the ones having $x$ component of the kinetic energy larger or smaller than $V_{0}$. 